Département de sciences économiques

2004-14

\title{
Nearly Serial Sharing Methods
}

SPRUMONT, Yves 


\section{Département de sciences économiques}

Université de Montréal

Faculté des arts et des sciences

C.P. 6128, succursale Centre-Ville

Montréal (Québec) H3C 3J7

Canada

http://www.sceco.umontreal.ca

SCECO-information@UMontreal.CA

Téléphone : (514) 343-6539

Télécopieur : (514) 343-7221

Ce cahier a également été publié par le Centre interuniversitaire de recherche en économie quantitative (CIREQ) sous le numéro 17-2004.

This working paper was also published by the Center for Interuniversity Research in Quantitative Economics (CIREQ), under number 17-2004.

ISSN 0709-9231 


\title{
Nearly Serial Sharing Methods
}

\author{
Yves SPRumont*
}

November 5, 2004

\begin{abstract}
A group of agents participate in a cooperative enterprise producing a single good. Each participant contributes a particular type of input; output is nondecreasing in these contributions. How should it be shared?

We analyze the implications of the axiom of Group Monotonicity: if a group of agents simultaneously decrease their input contributions, not all of them should receive a higher share of output. We show that in combination with other more familiar axioms, this condition pins down a very small class of methods, which we dub nearly serial.
\end{abstract}

$J E L$ classification numbers: C71, D63.

Keywords: surplus sharing, cost sharing, group monotonicity, serial method

\section{INTRODUCTION}

A group of agents participate in a cooperative enterprise producing a single good (which we may think of as money). Each participant contributes a possibly different type of input; total output is a nondecreasing function of these contributions. How should this total output (or gross profit) be shared? We search for a simple method that would compute output shares as a function of the profile of input contributions and the production function.

The case where input levels are either zero or one - each agent merely chooses whether to participate in the enterprise or not- corresponds to the model of cooperative games. In this much studied framework, the Shapley value (Shapley, 1953) stands out as the central sharing method. It is characterized by the three simple properties of Additivity, Dummy, and Anonymity: output shares depend additively on the production function, totally unproductive agents receive zero, and equally productive agents get the same share.

We are interested here in the more complex problem where each participant may choose any level of input contribution. Differences in output shares should now reflect not only the differences in productivity, as the Shapley value does in the simpler case, but also the differences in the levels of input contributions. For instance, if two agents are equally productive, the one who contributes a higher level of input should get a bigger share of output.

It is not surprising that in this richer model, Shapley's three axioms, properly reformulated, no longer characterize a unique method: there are different ways of combining the productivity and the sheer quantity of an input to compute the output share it deserves. Three prominent methods emerge from the literature. The Shapley-Shubik method (Shubik, 1962) applies the Shapley value to the so-called stand-alone game in which the worth of a coalition is the output generated by the input contributions of its members. The Aumann-Shapley method, as adapted from Aumann and Shapley (1974) by Billera and

*Département de Sciences Economiques and CIREQ, Université de Montréal, C.P. 6128, succursale centre-ville, Montréal H3C 3J7, Canada (yves.sprumont@umontreal.ca). I wrote a first draft of this paper while visiting CODE, Universitat Autònoma de Barcelona. Support from the Spanish Ministry of Education and the SSHRC of Canada is gratefully acknowledged. I thank H. Moulin for numerous enlighting conversations. 
Heath (1982) and Mirman and Tauman (1982), gives each agent the integral of his marginal product along the ray from zero to the input contribution profile. The serial method (Friedman and Moulin, 1999) integrates the marginal product of each agent along the constrained-diagonal path to the input contribution profile. ${ }^{1}$ We refer to Friedman and Moulin (1999) for a comparison of these three methods. Moulin (2002) offers a general survey, including a discussion of the (suitable reformulation of the) above methods in the case where inputs come in indivisible units; see also Moulin (1995).

In order to evaluate the relative merits of these and other methods, it is useful to formulate further axioms. An early example in the literature is Scale Invariance, stating that the output shares should not depend on the units of measurement of the inputs. This axiom is central in the classic characterizations of the Aumann-Shapley method (Billera and Heath, 1982, and Mirman and Tauman, 1982).

The very nature of the variable-input model suggests conditions linking the output shares in problems with different profiles of input contributions. Perhaps the simplest such condition is Monotonicity: a participant's share of output should be nondecreasing in his input contribution. Even though preferences are not an explicit component of our model, Monotonicity is readily interpreted as an incentive-compatibility condition. Any agent whose preferences are increasing in output and decreasing in the level of input supplied would have an incentive to manipulate a method that fails to be monotonic. Moulin (1995), who introduces the axiom, observes that it is satisfied by the ShapleyShubik and the serial methods but is violated by the Aumann-Shapley method.

Moulin and Sprumont (2004) strengthen Monotonicity by requiring that an increase in input contributions by a group of agents should not lead to a strict decrease in the output share of each of them. This is Group Monotonicity. A violation of this axiom leads to the possibility of strategic coordinated deflation of input contributions: all agents in some group could get a strictly higher share of output by agreeing to all reduce their contribution. In a cooperative environment where communication between agents is easy, Group Monotonicity is a compelling incentive-compatibility condition. Moulin and Sprumont (2004) show that the Shapley-Shubik method violates this condition, while the serial method satisfies it.

The purpose of this paper is to identify which methods meeting Shapley's three basic axioms satisfy Group Monotonicity. We work in the discrete version of the output-sharing model. Each agent $i$ contributes an integer level of input $x_{i}$; given the profile $x$ of all agents' contributions and the production function $F$, our method must allocate the total output $F(x)$.

The best known methods meeting Additivity and Dummy are the path methods. Fix an input profile $x$ and consider a sequence $\left\{z^{t}\right\}$ from zero to $x$ where each $z^{t}$ is obtained from its predecessor by increasing the input level of exactly one agent by one unit. For any production function $F$, compute any agent's output share by summing his marginal products along this sequence: agent $i$ thus receives $\sum\left(F\left(z^{t}\right)-F\left(z^{t-1}\right)\right)$ where the sum is taken over those $t$ for which $z^{t}$ obtains from $z^{t-1}$ by increasing $i$ 's contribution by one unit. Under the fixed-path methods, the paths used for different input profiles $x, x^{\prime}$ are related: they obtain by projection of a single unbounded path in input space. The socalled fixed-flow methods introduced in Moulin and Sprumont (2004) are essentially the

\footnotetext{
${ }^{1}$ The constrained-diagonal (or constrained-egalitaran) path to an input profile $x$ is the projection on $[0, x]$ of the diagonal path $z_{i}=z_{j}$ for all $i, j$. The method is called serial because it delivers the well-known serial formula of Moulin and Shenker $(1992,1994)$ when all inputs are perfect substitutes, that is, when total output depends only on the sum of the quantities supplied.
} 
convex combinations of fixed-path methods.

Our first result is an axiomatization of the latter methods. We use a new powerful variant of the Dummy axiom, Independence of Dummy Changes, stating that if the productivity of an agent is zero beyond a certain level of contribution, any input increase beyond that level should leave the output shares unchanged. Theorem 1 asserts that Additivity, Independence of Dummy Changes, and the mild requirement of Zero Output for Zero Input characterize the fixed-flow methods.

Building on this result, we then explore the implications of Group Monotonicity. As already mentioned, an important example of a group-monotonic method is the serial method. In our discrete model, all the unbounded paths staying as close as possible to the line $z_{i}=z_{j}$ for all $i, j$ qualify as "diagonal"; the serial method (Moulin, 1995) obtains by averaging the corresponding "diagonal" fixed-path methods. Theorem 2 states that the fixed-flow methods satisfying Anonymity and Group Monotonicity are, in a sense that will be made precise, nearly serial: they average fixed-path methods that are all nearly diagonal. Thus our axioms essentially characterize the serial method. We conjecture that an exact characterization holds in the continuous case.

A final word is in order about related contributions. Axiomatizations of various methods of the serial family exist in the literature. Moulin and Shenker (1994) characterize the serial formula for the case of perfectly substitutable inputs using an axiom placing upper bounds on output shares. With the aid of a similar axiom, Moulin (1995) and Friedman and Moulin (1999) characterize the serial method in the discrete and continuous contexts respectively. As noted in Moulin and Sprumont (2003), the Upper Bound axiom is intuitively reminiscent of the very serial formula. By contrast, none of the axioms used in Theorem 2 is directly related to a serial-type formula. Finally, Moulin and Sprumont (2003) offer an axiomatization of the serial method based on the property of Distributivity, which states that the sharing method should commute with the composition of production functions. This mathematical property akin to Additivity has no clear normative or strategic interpretation. By contrast, our main axiom, Group Monotonicity, is meaningful on both counts.

\section{THE MODEL}

Each agent $i$ in a finite set $N=\{1, \ldots, n\}$ contributes an integer quantity $x_{i} \in \mathbb{N}=\{0,1,2$, ... $\}$ of a personalized input. The output generated by the input profile $x \in \mathbb{N}^{N}$ must be split among the members of $N$. A production function is a mapping $F: \mathbb{N}^{N} \rightarrow \mathbb{R}_{+}$that is nondecreasing and satisfies $F(0)=0$; the set of such mappings is denoted $\mathcal{F}(N)$. A (output-sharing) method (for $N$ ) is a mapping $\varphi$ which assigns to each problem $(F, x) \in$ $\mathcal{F}(N) \times \mathbb{N}^{N}$ a vector of nonnegative output shares $\varphi(F, x) \in \mathbb{R}_{+}^{N}$ satisfying the budget balance condition $\sum_{i \in N} \varphi_{i}(F, x)=F(x)$.

An alternative interpretation of this model is to regard $x$ as a demand profile and $F$ as a cost function: $\varphi$ is then a cost-sharing method. We prefer the output-sharing interpretation because we find one of our axioms in Section 3, Independence of Dummy Changes, more convincing in that context.

We use the following notation. The set of extended natural numbers is $\overline{\mathbb{N}}=\mathbb{N} \cup\{+\infty\}$. Vector inequalities are written $\leq,<, \ll$. For any $x \in \mathbb{N}^{N}$ and $x^{\prime} \in \overline{\mathbb{N}}^{N},\left[x, x^{\prime}\right]=\{z \in$ $\left.\mathbb{N}^{N} \mid x \leq z \leq x^{\prime}\right\}$ and $\left.] x, x^{\prime}\right]=\left[x, x^{\prime}\right] \backslash\{x\}$. For any $S \subseteq N$, we denote by $x_{S} \in \mathbb{N}^{S}$ the restriction of $x$ to $S$ and write $x(S)=\sum_{i \in S} x_{i}$. We sometimes write $i$ for $\{i\}, i j$ for $\{i, j\}$, and $-S$ for $N \backslash S$. We define $e^{S} \in \mathbb{N}^{N}$ by $e_{i}^{S}=1$ if $i \in S$ and $e_{i}^{S}=0$ otherwise. If $i \in N$ and $F \in \mathcal{F}(N)$, we define $\partial_{i} F: \mathbb{N}^{N} \rightarrow \mathbb{R}_{+}$by $\partial_{i} F(z)=F(z)-F\left(z-e^{i}\right)$ whenever $z_{i}>0$ 
and, by convention, $\partial_{i} F(z)=0$ whenever $z_{i}=0$.

\section{The FIXED-FLOW METHODS}

This section proposes a simple axiomatization of the fixed-flow methods introduced in Moulin and Sprumont (2004). The first two axioms are well known, the third is new.

Additivity. For all $F, F^{\prime} \in \mathcal{F}(N)$ and $x \in \mathbb{N}^{N}, \varphi\left(F+F^{\prime}, x\right)=\varphi(F, x)+\varphi\left(F^{\prime}, x\right)$.

This powerful mathematical property is very convenient in practice. Consider a cooperative enterprise producing several goods which it sells on a market. The final good to be shared among theparticipants is money and the total amount is the sum of the sales of the different goods. One may apply the sharing method directly to the aggregated sales or use it to divide the sales of each good and then add up the resulting shares. If the method is additive, the two procedures are equivalent. This is useful from an incentive viewpoint because the proper level of application of the method is not a matter of dispute.

Our second axiom says that an agent who contributes nothing should receive nothing.

Zero Output for Zero Input. For all $F \in \mathcal{F}(N), x \in \mathbb{N}^{N}$, and $i \in N,\left\{x_{i}=0\right\} \Rightarrow$ $\left\{\varphi_{i}(F, x)=0\right\}$.

Our third axiom is inspired by the traditional Dummy axiom according to which a "dummy", that is, a completely unproductive agent, should receive zero: for all $F \in \mathcal{F}(N)$, $x \in \mathbb{N}^{N}$, and $i \in N,\left\{\partial_{i} F(z)=0\right.$ for all $\left.z \in \mathbb{N}^{N}\right\} \Rightarrow\left\{\varphi_{i}(F, x)=0\right\}$. Our condition says that "dummy changes" in input contributions should have no effect on output shares.

Irrelevance of Dummy Changes. For all $F \in \mathcal{F}(N), x \in \mathbb{N}^{N}$, and $i \in N,\left\{\partial_{i} F(z)=0\right.$ for all $z$ such that $\left.z_{i}>x_{i}\right\} \Rightarrow\left\{\varphi\left(F,\left(z_{i}, x_{-i}\right)\right)=\varphi(F, x)\right.$ for all $\left.z_{i}>x_{i}\right\}$.

Taken together, Zero Output for Zero Input and Irrelevance of Dummy Changes imply Dummy. In fact, they deliver a stronger property known as Strong Dummy: for all $F \in \mathcal{F}(N), x \in \mathbb{N}^{N}$, and $i \in N,\left\{\partial_{i} F(z)=0\right.$ for all $\left.z \in \mathbb{N}^{N}\right\} \Rightarrow\left\{\varphi_{i}(F, x)=0\right.$ and $\varphi_{j}(F, x)=\varphi_{j}\left(F,\left(0_{i}, x_{-i}\right)\right)$ for all $\left.j \in N \backslash i\right\}$. This says that a dummy agent gets zero and that his input contribution does not affect others' output shares.

In order to describe the methods meeting Additivity, Zero Output for Zero Input, and Irrelevance of Dummy Changes, we use Moulin and Vohra's (2003) characterization of the methods satisfying Additivity and Dummy.

Definition 1. A (unit) flow to an input profile $x \in \mathbb{N}^{N}$ is a mapping $f(., x):[0, x] \rightarrow \mathbb{R}_{+}^{N}$ such that $f_{i}(z, x)=0$ if $z_{i}=0, \sum_{i \in N(0, x)} f_{i}\left(e^{i}, x\right)=1$, and

$$
\left.\left.\sum_{i \in N} f_{i}(z, x)=\sum_{i \in N(z, x)} f_{i}\left(z+e^{i}, x\right) \text { for all } z \in\right] 0, x\right]
$$

where $N(z, x)=\left\{i \in N \mid z_{i}<x_{i}\right\}$. Conditions (1) are the so-called flow conservation constraints. A flow system is a list $f=\left\{f(., x) \mid x \in \mathbb{N}^{N}\right\}$, where each $f(., x)$ is a flow to $x$.

Moulin and Vohra (2003) prove that a method $\varphi$ satisfies Additivity and Dummy if and only if there is a flow system $f$ such that

$$
\varphi_{i}(F, x)=\sum_{z \in[0, x]} f_{i}(z, x) \partial_{i} F(z) \text { for all } F \in \mathcal{F}(N), x \in \mathbb{N}^{N}, \text { and } i \in N .
$$


This system $f$ is unique; we say that it represents $\varphi$. Notice that all such methods guarantee Zero Output for Zero Input. Observe also that the flows to different input profiles are totally unrelated.

We will be concerned with flow systems where the flow to an input profile is simply the projection of the flow to any higher input profile. For further use, we define the projection operator in full generality. Let $\underline{z} \in \mathbb{N}^{N}, \bar{z} \in \overline{\mathbb{N}}^{N}, \underline{z} \leq \bar{z}$. For any $T \subseteq N$, any mapping $h:[\underline{z}, \bar{z}] \rightarrow \mathbb{R}^{T}$ and any $x \in[\underline{z}, \bar{z}]$, the projection of $h$ on $[\underline{z}, x]$ is the mapping $p_{[\underline{z}, x]} h:[\underline{z}, x] \rightarrow \mathbb{R}^{T}$ defined as follows. For any $i \in T$ and $z \in[\underline{z}, x]$, write $K_{i}(z)=\left\{j \in N \backslash i \mid z_{j}=x_{j}\right\}$ and let

$$
\begin{aligned}
\left(p_{[\underline{z}, x]} h\right)_{i}(z) & =h_{i}(z) \text { if } K_{i}(z)=\emptyset, \\
& =\sum_{w_{K_{i}(z)} \in\left[x_{K_{i}(z)}, \bar{z}_{K_{i}(z)}\right]} h_{i}\left(w_{K_{i}(z)}, z_{-K_{i}(z)}\right) \text { otherwise. }
\end{aligned}
$$

Note that if $f(., x)$ is a flow to $x$ and $x^{\prime} \leq x$, then $p_{\left[0, x^{\prime}\right]} f(., x)$ is a flow to $x^{\prime}$.

Definition 2. A fixed flow system is a flow system $f$ such that

$$
\left\{x^{\prime} \leq x\right\} \Rightarrow\left\{f\left(., x^{\prime}\right)=p_{\left[0, x^{\prime}\right]} f(., x)\right\} \text { for all } x, x^{\prime} \in \mathbb{N}^{N} .
$$

A method $\varphi$ is a fixed-flow method if it is represented by a fixed flow system.

The fixed-flow methods were defined in Moulin and Sprumont (2004) under the restriction that input profiles are bounded above by some $\bar{x} \in \mathbb{N}^{N}$. In that case, the single flow $f(., \bar{x})$ and the projection property (3) fully determine the entire flow system $f$. In our unbounded version, the flow $f(., x)$ to any input profile $x$ completely determines the flows to all input profiles $x^{\prime} \leq x$. In particular, this implies that the system $f$ is completely determined by the subsystem $\left\{f\left(., k e^{N}\right) \mid k \in \mathbb{N}\right\}$.

Theorem 1. Let $N$ be an arbitrary nonempty finite set of agents. An output-sharing method $\varphi$ for $N$ satisfies Additivity, Zero Output for Zero Input, and Irrelevance of Dummy Changes if and only if $\varphi$ is a fixed-flow method.

Proof. The "if" statement is easily checked. To prove the converse statement, fix a method $\varphi$ meeting the three axioms. Because Zero Output for Zero Input and Irrelevance of Dummy Changes imply Dummy, the Moulin-Vohra representation theorem applies: let $f$ be the unique flow system representing $\varphi$ through formula (2). We show that $f$ is a fixed flow system.

In order to establish property (3), it is enough to prove that

$$
f(., x)=p_{[0, x]} f\left(., x+e^{i}\right) \text { for all } x \in \mathbb{N}^{N} \text { and } i \in N .
$$

This is because $p_{\left[0, x^{\prime \prime}\right]} p_{\left[0, x^{\prime}\right]} f(., x)=p_{\left[0, x^{\prime \prime}\right]} f(., x)$ whenever $x^{\prime \prime} \leq x^{\prime} \leq x$, as is clear from Definition 2.

The proof of (4) makes use of the following particular type of production function. For any $i \in N$ and $z \in \mathbb{N}^{N}$ such that $z_{i}>0$, we define $z_{0}^{j}:=z-z_{i} e^{i}+e^{j}$ if $j \in N \backslash i, z_{0}^{i}:=z$, and let

$$
F_{z}^{i}(w)=1 \text { if } w \geq z_{0}^{j} \text { for some } j \in N, \text { and } 0 \text { otherwise. }
$$

By construction,

$$
\partial_{i} F_{z}^{i}(w)=1 \text { if } w=z \text {, and } 0 \text { otherwise, }
$$


and

$$
\partial_{j} F_{z}^{i}(w)=0 \text { whenever } w_{j} \geq z_{j}+2 .
$$

We are now ready to prove (4). Fix $x \in \mathbb{N}^{N}$ and $i \in N$. The case $x=0$ being trivial, assume $x \neq 0$. We claim first that

$$
f_{i}(z, x)=f_{i}\left(z, x+e^{i}\right) \text { for all } z \in[0, x] .
$$

To see why this is true, fix $z \in[0, x]$. If $z_{i}=0, f_{i}(z, x)=f_{i}\left(z, x+e^{i}\right)=0$ by definition: so we may assume $z_{i}>0$. Consider the production function $F_{z}^{i}$. By definition, $\partial_{i} F_{z}^{i}(w)=0$ whenever $w_{i}>x_{i}$. Thus, by Irrelevance of Dummy Changes, $\varphi\left(F_{z}^{i}, x+e^{i}\right)=\varphi\left(F_{z}^{i}, x\right)$. Focusing on agent $i$ 's output share and using (2) and (6), $f_{i}(z, x)=\varphi_{i}\left(F_{z}^{i}, x\right)=\varphi_{i}\left(F_{z}^{i}, x+\right.$ $\left.e^{i}\right)=f_{i}\left(z, x+e^{i}\right)$.

Next we claim that

$$
f_{j}(z, x)=f_{j}\left(z, x+e^{i}\right) \text { for all } j \in N \backslash i \text { and all } z \in[0, x] \text { such that } z_{i}<x_{i} .
$$

Fix $j \in N \backslash i$ and $z \in[0, x]$ such that $z_{i}<x_{i}$. If $z_{j}=0, f_{j}(z, x)=f_{j}\left(z, x+e^{i}\right)=0$, so assume $z_{j}>0$. Consider the production function $F_{z}^{j}$. By definition, $\partial_{i} F_{z}^{j}(w)=0$ whenever $w_{i} \geq z_{i}+2$. Since $z_{i}<x_{i}, \partial_{i} F_{z}^{j}(w)=0$ whenever $w_{i}>x_{i}$. By Irrelevance of Dummy Changes, $\varphi\left(F_{z}^{j}, x+e^{i}\right)=\varphi\left(F_{z}^{j}, x\right)$. Focusing on agent $j$ and using the representation formula $(2), f_{j}(z, x)=\varphi_{j}\left(F_{z}^{j}, x\right)=\varphi_{j}\left(F_{z}^{j}, x+e^{i}\right)=f_{j}\left(z, x+e^{i}\right)$.

To complete the proof of (4), it remains to be shown that

$f_{j}(z, x)=f_{j}\left(z, x+e^{i}\right)+f_{j}\left(z+e^{i}, x+e^{i}\right)$ for all $j \in N \backslash i$ and all $z \in[0, x]$ such that $z_{i}=x_{i}$.

Fix $j \in N \backslash i$ and $z \in[0, x]$ such that $z_{i}=x_{i}$. Let $F$ be the production function such that

$$
\begin{aligned}
& \partial_{i} F(w)=0 \text { for all } w \in \mathbb{N}^{N}, \text { and } \\
& \partial_{j} F(w)=1 \text { if } w_{-i}=z_{-i}, \text { and } 0 \text { otherwise. }
\end{aligned}
$$

This production function is easily constructed: define it first on those input profiles such that $z_{i}=0$ by modifying the procedure in (5), then extend it to all profiles by setting $F(z)=F\left(0_{i}, z_{-i}\right)$. By Irrelevance of Dummy Changes, $\varphi_{j}\left(F, x+e^{i}\right)=\varphi_{j}(F, x)$. Applying the representation formula $(2)$,

$$
\begin{aligned}
\varphi_{j}(F, x) & =\sum_{w_{i}=0}^{x_{i}} f_{j}\left(\left(w_{i}, z_{-i}\right), x\right), \\
\varphi_{j}\left(F, x+e^{i}\right) & =\sum_{w_{i}=0}^{x_{i}+1} f_{j}\left(\left(w_{i}, z_{-i}\right), x+e^{i}\right) .
\end{aligned}
$$

Taking (9) into account, this means that $f_{j}\left(\left(x_{i}, z_{-i}\right), x\right)=f_{j}\left(\left(x_{i}, z_{-i}\right), x+e^{i}\right)+f_{j}\left(\left(x_{i}+\right.\right.$ $\left.\left.1, z_{-i}\right), x+e^{i}\right)$. Recalling that $z_{i}=x_{i}$, this means $f_{j}(z, x)=f_{j}\left(z, x+e^{i}\right)+f_{j}\left(z+e^{i}, x+\right.$ $\left.e^{i}\right)$.

The axioms in Theorem 1 are independent. This follows directly from the independence of the axioms used in Theorem 2, which is established in the next section. 


\section{THE NEARLY SERIAL METHODS}

Building on the previous section, we now show how two further axioms, Anonymity and Group Monotonicity, circumscribe the very small subclass of fixed-flow methods that we call nearly serial.

We begin by defining Anonymity. Denote by $\Pi(N)$ the set of bijections from $N$ into itself and let $\pi \in \Pi(N)$. If $z \in \mathbb{R}_{+}^{N}$, define $\pi z \in \mathbb{R}_{+}^{N}$ by $(\pi z)_{\pi(i)}=z_{i}$ for all $i \in N$. If $F \in \mathcal{F}(N)$, define $\pi F \in \mathcal{F}(N)$ by $\pi F(\pi z)=F(z)$ for all $z \in \mathbb{N}^{N}$.

Anonymity. For all $\pi \in \Pi(N), F \in \mathcal{F}(N)$, and $x \in \mathbb{N}^{N}, \varphi(\pi F, \pi x)=\pi \varphi(F, x)$.

This axiom expresses the familiar idea that the names of the agents should be ignored when computing the output shares: it is generally accepted as a basic notion of fairness. Our formulation implies weaker symmetry properties used in the literature: for instance, agents with equal input contributions receive the same output share when the production function is symmetric.

A method $\varphi$ satisfying Additivity and Dummy meets Anonymity if and only if its flow system $f$ satisfies $f(\pi z, \pi x)=\pi f(z, x)$ for all $x \in \mathbb{N}^{N}, z \in[0, x]$, and $\pi \in \Pi(N)$. Sufficiency is clear from the representation formula (2). For a proof of necessity, suppose $\varphi$ meets Anonymity and fix $x, z$, and $\pi$ as required. Let $i \in N$ and consider the production function $F_{z}^{i}$ defined in (5). By (2) and Anonymity, $f_{i}(z, x)=\varphi_{i}(F, x)=\varphi_{\pi(i)}(\pi F, \pi x)=$ $f_{\pi(i)}(\pi z, \pi x)$, as claimed.

Focusing now on fixed-flow methods, let $k \in \mathbb{N}$ and consider the input profile $k e^{N}$ where all agents contribute $k$ units. We say that a flow $f\left(., k e^{N}\right)$ to $k e^{N}$ is anonymous if

$$
f\left(\pi z, k e^{N}\right)=\pi f\left(z, k e^{N}\right) \text { for all } z \in\left[0, k e^{N}\right] \text { and } \pi \in \Pi(N) .
$$

Taking into account the remark following Definition 2, a fixed-flow method $\varphi$ meets Anonymity if and only if each flow $f\left(., k e^{N}\right), k \in \mathbb{N}$, is anonymous. We then say that the fixed flow system $f$ is anonymous.

We introduce now the serial and nearly serial methods. In order to do so, we need some further definitions.

Definition 3. Let $D=\left\{z \in \mathbb{N}^{N}|| z_{i}-\frac{z(N)}{n} \mid<1\right.$ for all $\left.i \in N\right\}$ and $\bar{D}=\left\{z \in \mathbb{N}^{N} \mid\right.$ $\left|z_{i}-\frac{z(N)}{n}\right| \leq 1$ for all $\left.i \in N\right\}$; we call these sets the diagonal and near-diagonal of $\mathbb{N}^{N}$. If $f(., x)$ is a flow to an input profile $x \in \mathbb{N}^{N}$, the set $S(f, x)=\{z \in[0, x] \mid f(z, x) \neq 0\}$ is called the support of $f(., x)$. A flow $f\left(., k e^{N}\right)$ is diagonal if $S\left(f, k e^{N}\right) \subseteq D$, and nearly diagonal if $S\left(f, k e^{N}\right) \subseteq \bar{D}$. A fixed flow system $f$ is (nearly) diagonal if $f\left(., k e^{N}\right)$ is (nearly) diagonal for all $k \in \mathbb{N}$.

A quick description of $D$ and $\bar{D}$ may be useful. For each $t \in \mathbb{N}$, define $\Delta(t)=\left\{z \in \mathbb{N}^{N} \mid\right.$ $z(N)=t\}$ and let $D(t)=D \cap \Delta(t)$ and $\bar{D}(t)=\bar{D} \cap \Delta(t)$.

If $t$ is a multiple of $n$, say $t=r n$ for some $r \in \mathbb{N}$, then $D(t)=\left\{r e^{N}\right\}$. If $t=r n+1$ for some $r \in \mathbb{N}, D(t)$ is made up of all permutations of the point $(r+1, r, \ldots, r)$. More generally, if $t=r n+k$ for some $r \in \mathbb{N}$ and $k \in\{1, \ldots, n-1\}, D(t)$ is made up of all permutations of the point $(r+1) e^{\{1, \ldots, k\}}+r e^{\{k+1, \ldots, n\}}$.

The set $\bar{D}(t)$ coincides with $D(t)$ whenever $t$ is not a positive multiple of $n$. If $t$ is a positive multiple of $n$, say $t=n r$ for some $r \in \mathbb{N} \backslash\{0\}$, then $\bar{D}(t)$ is larger than $D(t)$. It contains all points that can be written as $(r+1) e^{\{1, \ldots k\}}+r e^{\{k+1, \ldots, n-k\}}+(r-$ 1) $e^{\{n-k+1, \ldots, n\}}$ for some integer $k, 0 \leq k \leq \frac{n}{2}$ (with the understanding that $e^{\emptyset}=0$ ), and all their permutations. 
Figures 1 and 2 illustrate $D$ and $\bar{D}$ when $n=2$ and 3 respectively.

It is well known that there is only one anonymous flow to the one-unit-contribution profile $e^{N}$, namely

$$
f_{i}^{S}\left(z, e^{N}\right)=\frac{n_{i}(z) !\left(n-n_{i}(z)-1\right) !}{n !} \text { for all } i \in N \text { such that } z_{i}=1,
$$

where $n_{i}(z)=\left|\left\{j \in N \backslash i \mid z_{j}=1\right\}\right|$. As an illustration, Figure 3 depicts $f^{S}\left(., e^{\{1,2,3\}}\right)$. On the subset $\left\{\left(F, e^{N}\right) \mid F \in \mathcal{F}(N)\right\}$ (that is, on the problems corresponding to standard cooperative games), the flow $f^{S}\left(., e^{N}\right)$ generates the Shapley value.

Next, fix $k \in \mathbb{N}$. Because the support of a diagonal flow to $k e^{N}$ is included in the union of the unit cubes $\left[0, e^{N}\right],\left[e^{N}, 2 e^{N}\right], \ldots,\left[(k-1) e^{N}, k e^{N}\right]$, there is a unique anonymous diagonal flow to $k e^{N}$ : we denote it $f^{s}\left(., k e^{N}\right)$. Figure 4 depicts $f^{s}\left(., 2 e^{\{1,2,3\}}\right)$. Recalling the remarks following Definition 2, the subsystem $\left\{f^{s}\left(., k e^{N}\right) \mid k \in \mathbb{N}\right\}$ completely determines a unique fixed flow system, which we denote $f^{s}$.

Definition 4. The serial method $\varphi^{s}$ is the output-sharing method represented by the unique anonymous diagonal fixed flow system $f^{s}$.

This method was proposed by Moulin (1995). When all inputs are perfect substitutes, that is, when the production function takes the form $F(z)=\widetilde{F}(z(N))$ for some function $\widetilde{F}$ from $\mathbb{N}$ to $\mathbb{R}_{+}$, it reduces to the well-known serial mechanism (Moulin and Shenker, 1992): assuming without loss of generality that $x_{1} \leq x_{2} \leq \ldots \leq x_{n}$, the output shares are $\varphi_{1}(F, x)=\frac{1}{n} \widetilde{F}\left(n x_{1}\right), \varphi_{2}(F, x)=\frac{1}{n} \widetilde{F}\left(n x_{1}\right)+\frac{1}{n-1}\left[\widetilde{F}\left(x_{1}+(n-1) x_{2}\right)-\widetilde{F}\left(n x_{1}\right)\right], \ldots$, $\varphi_{n}(F, x)=\frac{1}{n} \widetilde{F}\left(n x_{1}\right)+\frac{1}{n-1}\left[\widetilde{F}\left(x_{1}+(n-1) x_{2}\right)-\widetilde{F}\left(n x_{1}\right)\right]+\ldots+\left[\widetilde{F}\left(x_{1}+x_{2}+\ldots+x_{n}\right)-\right.$ $\left.\widetilde{F}\left(x_{1}+x_{2}+\ldots+x_{n-2}+2 x_{n-1}\right)\right]$.

The methods we will characterize are only slight variations of the serial method.

Definition 5. A nearly serial method is an output-sharing method represented by an anonymous nearly diagonal fixed flow system.

While there are several anonymous nearly diagonal fixed flow systems, they form a rather small family. Each such system $f$ is conveniently represented by a single anonymous nearly diagonal unbounded flow, that is, a mapping $\bar{f}: \mathbb{N}^{N} \rightarrow \mathbb{R}_{+}^{N}$ such that $\bar{f}_{i}(z)=0$ if $z \notin \bar{D}$ or $z-e^{i} \notin \bar{D}, \sum_{i \in N} \bar{f}_{i}\left(e^{i}\right)=1$,

$$
\sum_{i \in N} \bar{f}_{i}(z)=\sum_{i \in N} \bar{f}_{i}\left(z+e^{i}\right) \text { for all } z \in \bar{D} \backslash\{0\},
$$

and

$$
\bar{f}(\pi z)=\pi \bar{f}(z) \text { for all } z \in \bar{D} .
$$

For each $x \in \mathbb{R}^{N}, f(., x)=p_{[0, x]} \bar{f}$. We let $S(\bar{f})=\left\{z \in \mathbb{N}^{N} \mid \bar{f}(z) \neq 0\right\}$.

A first important observation is that $\bar{D}(t)$ is a perfectly symmetric set whenever $t$ is not a multiple of $n$ : each element of $\bar{D}(t)$ is a permutation of any other element of $\bar{D}(t)$. Therefore, the anonymity condition (12) implies that, for any $t$ that is not a positive multiple of $n, S(\bar{f}) \cap \bar{\Delta}(t)=\bar{\Delta}(t)$, and $\bar{f}$ is completely determined on $\bar{\Delta}(t)$ once it is determined on $\bar{\Delta}(t-1)$.

A second observation is that $S(\bar{f}) \cap \bar{\Delta}(t)$ is necessarily a rather small subset of $\bar{D}(t)$ when $t$ is a positive multiple of $n$. In fact, for all $r \in \mathbb{N} \backslash\{0\}$ and all $z \in \bar{D}(r n)$,

$$
[\bar{f}(z) \neq 0] \Rightarrow\left[z=r e^{N} \text { or } z \text { is a permutation of }(r-1) e^{1}+r e^{\{2, . ., n-1\}}+(r+1) e^{n}\right] .
$$


This follows immediately from the flow conservation constraints (11). So $S(\bar{f}) \cap \bar{\Delta}(r n)$ is the union of a singleton and a perfectly symmetric set.

These two observations imply that $\bar{f}$ is fully characterized by a sequence $\left\{\alpha_{r}\right\}_{r \in \mathbb{N} \backslash\{0\}}$ in $[0,1]$, where

$$
\alpha_{r}=\sum_{i \in N} \bar{f}_{i}\left(r e^{N}\right)
$$

is the fraction of the total (unit) flow that goes through $r e^{N}$. This is illustrated in Figure $\mathbf{5}$ for $n=2$ and in Figures 6.a and 6.b for $n=3$. (Note that $S(\bar{f}) \cap \bar{\Delta}(r n)$ is a strict subset of $\bar{\Delta}(r n)$ only when $n \geq 4$.) Choosing $\alpha_{r}=1$ for all $r$ guarantees that $\bar{f}$ is in fact diagonal, that is, $S(\bar{f}) \subseteq D$, and generates the serial method.

We now come to our central axiom and our main result. We ask that when a group of agents increase their input contributions, not all of them get less output.

Group Monotonicity. For all $F \in \mathcal{F}(N)$, all $x, x^{\prime} \in \mathbb{N}^{N}$, and all nonempty $S \subseteq N$, $\left\{x_{i}<x_{i}^{\prime}\right.$ for all $i \in S$ and $x_{i}=x_{i}^{\prime}$ for all $\left.i \in N \backslash S\right\} \Rightarrow$ there exists $i \in S$ such that $\left.\varphi_{i}(F, x) \leq \varphi_{i}\left(F, x^{\prime}\right)\right\}$.

This property is a natural generalization of the standard Monotonicity axiom, which corresponds to the case where $S$ is a singleton. As discussed in the Introduction, Group Monotonicity is best defended from the strategic angle: it prevents coordinated deflation of input contributions.

Theorem 2. Let $N$ be a finite set containing at least three agents. An output-sharing method $\varphi$ for $N$ satisfies Additivity, Zero Output for Zero Input, Irrelevance of Dummy Changes, Anonymity, and Group Monotonicity if and only if $\varphi$ is a nearly serial method.

\section{Proof.}

The rather tedious proof of the "if" statement is relegated to the Appendix. In order to prove the "only if" statement, let $N=\{1,2, \ldots, n\}$ be a set of agents, $n \geq 3$. Let $\varphi$ be an output-sharing method for $N$ meeting all five axioms in Theorem 2. Recalling Theorem 1 and the remarks following the definition of Anonymity, $\varphi$ is represented by a unique anonymous fixed flow system $f$. We will prove by induction on $n$ that $f$ is nearly diagonal.

Step 1. $f$ is nearly diagonal if $n=3$.

We fix $N=\{1,2,3\}$ and claim that $S\left(f, k e^{N}\right) \subseteq \bar{D}$ for all $k \in \mathbb{N} \backslash\{0\}$. To prove our claim its suffices to show

$$
\forall k \in \mathbb{N} \backslash\{0\}, \forall t \in \mathbb{N} \backslash\{0\} \text { such that } t \leq k, S\left(f, k e^{N}\right) \cap \Delta(t) \subseteq \bar{D}(t) .
$$

(Indeed, let $k \in \mathbb{N} \backslash\{0\}$ and suppose $z \in S\left(f, k e^{N}\right) \subseteq \bar{D}$. Set $t:=z(N)$. If $t \leq k$, (14) implies $z \in \bar{D}$, as desired. If $t>k$, simply choose $k^{\prime} \geq t>k$. By the fixed-flow property, $z \in S\left(f, k^{\prime} e^{N}\right)$, so that (14) implies $S\left(f, k^{\prime} e^{N}\right) \cap \Delta(t) \subseteq \bar{D}(t)$, hence $z \in \bar{D}$ again.)

From now on, we fix $k \in \mathbb{N} \backslash\{0\}$ and (with a slight abuse of notation) write $f$ instead of $f\left(., k e^{N}\right)$. We show by induction on $t$ that $S(f) \cap \Delta(t) \subseteq \bar{D}(t)$ for all $t \in \mathbb{N} \backslash\{0\}$.

If $t=1$, the anonymity of $f$ directly implies that

$$
S(f) \cap \Delta(1)=\bar{D}(1) .
$$

Next we fix $t, 2 \leq t \leq k$, assume $S(f) \cap \Delta(\tau) \subseteq \bar{D}(\tau)$ for $\tau=1, \ldots, t-1$, and show that $S(f) \cap \Delta(t)=\bar{D}(t)$. Recall that the set $\bar{D}(t)$ may be of three different types depending 
on the value of $t$ :

$$
\begin{aligned}
\bar{D}(t)= & \{(r, r, r+1),(r, r+1, r),(r+1, r, r)\} \\
\text { if } t= & 3 r+1 \text { for some } r \in \mathbb{N} \backslash\{0\}, \\
\bar{D}(t)= & \{(r, r+1, r+1),(r+1, r, r+1),(r+1, r+1, r)\} \\
\text { if } t= & 3 r+2 \text { for some } r \in \mathbb{N}, \\
\bar{D}(t)= & \{(r, r, r),(r-1, r, r+1),(r-1, r+1, r),(r, r-1, r+1), \\
& (r, r+1, r-1),(r+1, r-1, r),(r+1, r, r-1)\}
\end{aligned}
$$

if $t=3 r$ for some $r \in \mathbb{N} \backslash\{0\}$.

We deal with each type separately. Given $z \in \mathbb{N}^{N} \backslash\{0\}$, we call $f(z)=\left(f_{1}(z), f_{2}(z), f_{3}(z)\right)$ the flow at $z$. By the flow at a set $Z$ we mean the collection $\{f(z) \mid z \in Z\}$.

Case 1: $t=3 r+1$ for some $r \in \mathbb{N} \backslash\{0\}$.

By the induction hypothesis, $S(f) \cap \Delta(3 r) \subseteq \bar{D}(3 r)$. The latter set is made up of 7 points: $(r, r, r)$ and the 6 permutations of $(r-1, r, r+1)$. The induction hypothesis also ensures that $S(f) \cap \Delta(3 r-1) \subseteq \bar{D}(3 r-1)$, which is made up of the three permutations of $(r-1, r, r)$. By Anonymity of $f$, the flow at $\bar{D}(3 r)$ is therefore determined up to one parameter $\alpha \in[0,1]$ :

$$
\begin{aligned}
f(r, r, r) & =\left(\frac{\alpha}{3}, \frac{\alpha}{3}, \frac{\alpha}{3}\right), \\
f(r-1, r, r+1) & =\left(0,0, \frac{1-\alpha}{6}\right),
\end{aligned}
$$

and the flow at each permutation of $(r-1, r, r+1)$ obtains by applying that permutation to $f(r-1, r, r+1)$. See Figure 7 .

We want to show that

$$
S(f) \cap \Delta(3 r+1) \subseteq \bar{D}(3 r+1),
$$

where $\bar{D}(3 r+1)$ is made up of the three permutations of $(r, r, r+1)$. By the induction hypothesis, flow conservation and the anonymity of $f$, it suffices to show that

$$
f_{1}(r+1, r+1, r-1)=0
$$

and

$$
f_{1}(r+2, r, r-1)=0 .
$$

The flow at $\bar{D}(3 r+1)$ is then fully determined by flow conservation: $f(r+1, r, r)=$ $\left(\frac{\alpha}{3}, \frac{1-\alpha}{6}, \frac{1-\alpha}{6}\right)$ and the flow at the permuted points obtains by permutation. See Figure 8.

To prove (15), suppose, by way of contradiction, $f_{1}(r+1, r+1, r-1)=f_{2}(r+1, r+1, r-$ $1)=\beta, 0<\beta \leq \frac{1-\alpha}{6}$. Write $g=f(.,(r+1, r+1, r+1))$, the projection of $f$ on $\left[0,(r+1) e^{N}\right]$, and note that $g$ is fully determined. In particular, $g(r+1, r-1, r+1)=(\beta, 0, \beta)$, $g(r-1, r+1, r+1)=(0, \beta, \beta)$, and $g(r, r, r+1)=\left(\frac{1-\alpha}{6}-\beta, \frac{1-\alpha}{6}-\beta, \frac{\alpha}{3}\right)$. Define the production function $F$ by $F(w)=1$ if $w \geq z$ for some $z \in\{(r, r, r+1),(r+1, r-$ $1, r+1),(r-1, r+1, r+1)\}$ and $F(w)=0$ otherwise. Then $\varphi_{1}(F,(r+1, r+1, r+1))=$ $\varphi_{2}(F,(r+1, r+1, r+1))=\left(\frac{1-\alpha}{6}-\beta\right)+\beta=\frac{1-\alpha}{6}$. But using the fixed-flow property, compute now $\varphi_{1}(F,(r, r, r+1))=g_{1}(r, r, r+1)+g_{1}(r, r+1, r+1)=\left(\frac{1-\alpha}{6}-\beta\right)+2 \beta=\frac{1-\alpha}{6}+\beta$, 
which is also $\varphi_{2}(F,(r, r, r+1))$ by Anonymity. This violates Group Monotonicity. See Figure 9.

We have proved (15). In fact, we have shown more, namely $g_{1}(r+1, r+1, r-1)(=$ $\left.g_{2}(r+1, r+1, r-1)\right)=0$. As a consequence, by flow conservation,

$$
g_{3}(r+1, r+1, r)=0 .
$$

In order to prove (16), suppose $f_{1}(r+2, r, r-1)=f_{2}(r, r+2, r-1)=\gamma, 0<\gamma \leq \frac{1-\alpha}{6}$. Define the production function $F^{\prime}$ by $F^{\prime}(w)=1$ if $w \geq z$ for some $z \in\{(r+1, r+1, r),(r+$ $2, r, r),(r, r+2, r)\}$ and $F(w)=0$ otherwise. See Figure 10. Then $\varphi_{3}\left(F^{\prime},(r+1, r+\right.$ $1, r+1))=g_{3}(r+1, r+1, r)=0$. On the other hand, by the fixed-flow property and flow conservation, $\varphi_{3}\left(F^{\prime},(r+2, r+2, r+1)\right) \geq \varphi_{3}\left(F^{\prime},(r+2, r+2, r)\right)=2 \gamma>0$. Using Anonymity, $\varphi_{i}\left(F^{\prime},(r+1, r+1, r+1)\right)>\varphi_{i}\left(F^{\prime},(r+2, r+2, r+1)\right)$ for $i=1,2$, violating Group Monotonicity.

Case 2: $t=3 r+2$ for some $r \in \mathbb{N}$.

By the induction hypothesis, $S(f) \cap \Delta(3 r+1) \subseteq \bar{D}(3 r+1)$ and by anonymity of $f$, the flow at $\bar{D}(3 r+1)$ is as shown in Figure 8: $f(r+1, r, r)=\left(\frac{\alpha}{3}, \frac{1-\alpha}{6}, \frac{1-\alpha}{6}\right)$ and the the flow at the permuted points obtains by permutation. We claim now that

$$
S(f) \cap \Delta(3 r+2) \subseteq \bar{D}(3 r+2) .
$$

Because of the anonymity of $f$, we need only show

$$
f_{1}(r+2, r, r)=0 .
$$

Suppose $f_{1}(r+2, r, r)=\delta, 0<\delta \leq \frac{1}{3}$. Define the production function $F^{\prime \prime}$ by $F^{\prime \prime}(w)=1$ if $w \geq z$ for some $z \in\{(r+1, r+1, r+1),(r+2, r, r+1),(r, r+2, r+1)\}$ and $F(w)=0$ otherwise, as shown in Figure 11. By Anonymity, $\varphi_{3}\left(F^{\prime \prime},(r+1, r+1, r+1)\right)=\frac{1}{3}$. On the other hand, by the fixed-flow property and flow conservation, $\varphi_{3}\left(F^{\prime \prime},(r+2, r+2, r+1)\right) \geq$ $\delta+\left(\frac{1}{6}-\frac{\delta}{2}\right)+\left(\frac{1}{6}-\frac{\delta}{2}\right)+\delta=\frac{1}{3}+\delta>\frac{1}{3}$. So $\varphi_{i}\left(F^{\prime \prime},(r+1, r+1, r+1)\right)>\varphi_{i}\left(F^{\prime \prime},(r+2, r+2, r+1)\right)$ for $i=1,2$, contradicting Group Monotonicity. This proves (17). Note that the anonymity of $f$ completely determines the entire flow at $\bar{D}(3 r+2): f_{1}(r+1, r+1, r)=f_{2}(r+1, r+$ $1, r)=f_{1}(r+1, r, r+1)=f_{3}(r+1, r, r+1)=f_{2}(r, r+1, r+1)=f_{3}(r, r+1, r+1)=\frac{1}{6}$, as depicted on Figure 12.

Case 3: $t=3 r$ for some $r \in \mathbb{N} \backslash\{0\}$.

By the induction hypothesis, $S(f) \cap \Delta(3 r-1) \subseteq \bar{D}(3 r-1)$ (and the flow at $\bar{D}(3 r-1)$ is as shown on Figure 13). It follows directly from flow conservation that $S(f) \cap \Delta(3 r) \subseteq$ $\bar{D}(3 r)$. Moreover, by anonymity of $f$, the flow at $\bar{D}(3 r)$ must be as shown on Figure 7 : $f(r, r, r)=\left(\frac{\alpha}{3}, \frac{\alpha}{3}, \frac{\alpha}{3}\right), f(r-1, r, r+1)=\left(0,0, \frac{1-\alpha}{6}\right)$, and the flow at each permutation of $(r-1, r, r+1)$ obtains by applying that permutation to $f(r+1, r, r-1)$.

Step 2. Induction argument.

Let $N=\{1, \ldots, n\}, n>3$. Make the induction hypothesis that that for every $M \subseteq N$ such that $3 \leq|M|<n$, every output-sharing method for $M$ satisfying our five axioms is a nearly serial method. Let $\varphi$ be an output-sharing method for $N$ satisfying the five axioms. Let $f$ be the flow system representing $\varphi$. By Theorem 1 and the comments following the definition of Anonymity, $f$ is an anonymous fixed flow system. We must prove that it is nearly diagonal. 
Suppose, by contradiction, that there exists $k>0, z \in S\left(f, k e^{N}\right)$, and $i \in N$ such that

$$
\left|z_{i}-\frac{z(N)}{n}\right|>1
$$

We will show that

$$
\left|z_{i}-\frac{z(N \backslash j)}{n-1}\right|>1 \text { for some } j \in N \backslash i .
$$

To see that this yields a contradiction, define the system $f^{-j}=\left\{f^{-j}\left(., x_{N \backslash j}\right) \mid x_{N \backslash j} \in\right.$

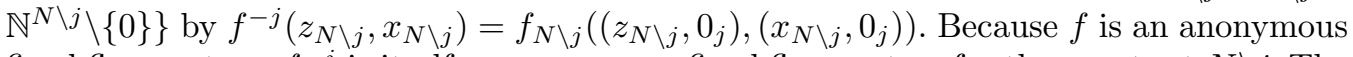
fixed flow system, $f^{-j}$ is itself an anonymous fixed flow system for the agent set $N \backslash j$. The output-sharing method $\varphi^{-j}$ for $N \backslash j$ that $f^{-j}$ represents satisfies

$$
\varphi^{-j}\left(F^{-j}, x_{N \backslash j}\right)=\varphi_{N \backslash j}\left(F,\left(x_{N \backslash j}, 0_{j}\right)\right)
$$

for all $x_{N \backslash j} \in \mathbb{N}^{N \backslash j}$ and $F \in \mathcal{F}(N)$, where $F^{-j}\left(z_{N \backslash j}\right):=F\left(z_{N \backslash j}, 0_{j}\right)$. Since $\varphi$ satisfies Group Monotonicity, so does $\varphi^{-j}$. Because $f$ is a fixed flow system, $z \in S\left(f, k e^{N}\right)$ implies $\left(z_{N \backslash j}, 0_{j}\right) \in S\left(f, k e^{N \backslash j}\right)$. Hence, by definition of $\varphi^{-j}$,

$$
z_{N \backslash j} \in S\left(f^{-j}, k e^{N \backslash j}\right),
$$

and (19) and (20) contradict the induction hypothesis.

Now we prove (19). Starting from (18), distinguish two cases: either $z_{i}-\frac{z(N)}{n}>1$ or $\frac{z(N)}{n}-z_{i}>1$. We only consider the former case, the latter is similar and left to the reader. Without loss of generality, assume $i=1$, so that

$$
(n-1) z_{1}>z(N \backslash 1)+n .
$$

Assume without loss of generality

$$
z_{1} \geq z_{2} \geq \ldots \geq z_{n}
$$

In order to prove (19), it suffices to show that

$$
(n-2) z_{1}>z(N \backslash\{1,2\})+(n-1) .
$$

Suppose, by contradiction, that

$$
(n-2) z_{1} \leq z(N \backslash\{1,2\})+(n-1) .
$$

Then $(n-1) \leq z(N \backslash 2)+(n-1)$ and therefore, by $(21), z(N \backslash 1)+n<z(N \backslash 2)+(n-1)$. Hence,

$$
z_{2}+1<z_{1}
$$

By $(22),\left(z_{3}+1\right), \ldots,\left(z_{n}+1\right)<z_{1}$, hence $(n-2) z_{1}>z(N \backslash\{1,2\})+(n-2)$, which, combined with (23), gives $(n-2) z_{1}=z(N \backslash\{1,2\})+(n-1)$. Using (24),

$$
z_{2}<\frac{z(N \backslash\{1,2\})+1}{n-2} .
$$

If at least one inequality is strict in the string $z_{3} \geq z_{4} \geq \ldots \geq z_{n}$, then $z_{n}+1 \leq z_{3}$ and it follows that $z(N \backslash\{1,2\})+1 \leq(n-2) z_{3}$. Therefore (25) implies $z_{2}<z_{3}$, a contradiction. 
If $z_{3}=z_{4}=\ldots=z_{n}$, then (25) reads $z_{2}<z_{3}+\frac{1}{n-2}$ and, because of (22), $z_{2}=z_{3}$. Thus (23) becomes $(n-2) z_{1} \leq(n-2) z_{2}+(n-1)$, hence $z_{1} \leq z_{2}+\frac{n-1}{n-2}<z_{2}+2$ and, since $z_{1}, z_{2}$ are integers, $z_{1} \leq z_{2}+1$. This contradicts (24).

We conclude with three comments.

1) It is easy to see that the axioms in Theorem 2 are independent. For a method satisfying all axioms but Additivity, consider the following adjusted proportional method. Given a problem $(F, x)$, define $a_{i F}^{*}=\min \left\{a_{i} \mid \partial_{i} F(z)=0\right.$ for all $z$ such that $\left.z_{i} \geq a_{i}\right\}$ (with the convention that $a_{i F}^{*}=+\infty$ if the latter set is empty) and let $x_{i F}^{*}=\min \left\{x_{i}, a_{i F}^{*}\right\}$. Define $\varphi_{i}(F, x)=\frac{x_{i F}^{*}}{x_{F}^{*}(N)} F(x)$ if $x_{F}^{*}(N)>0$ (and $\varphi(F, x)=0$ if $\left.x_{F}^{*}(N)=0\right)$. An example violating only Zero Output for Zero Input is the egalitarian method $\varphi_{i}(F, x)=\frac{1}{n} F(x)$. A method violating only Independence of Dummy Changes is the proportional method $\varphi_{i}(F, x)=\frac{x_{i}}{x(N)} F(x)$ if $x(N)>0$ (and $\varphi(F, 0)=0$ ). For an example violating only Anonymity, consider any fixed-path method. Finally, the Shapley-Shubik method satisfies all axioms but Group Monotonicity. See Moulin and Sprumont (2004) for details.

2) The proof does not use the full force of Group Monotonicity. That axiom may be replaced in Theorem 2 by the following weaker requirement.

Pair Monotonicity. For all $F \in \mathcal{F}(N)$, all $x, x^{\prime} \in \mathbb{N}^{N}$, and all pairs of distinct agents $i, j \in N,\left\{x_{i}<x_{i}^{\prime}, x_{j}<x_{j}^{\prime}\right.$, and $x_{k}=x_{k}^{\prime}$ for all $\left.k \in N \backslash i j\right\} \Rightarrow\left\{\varphi_{i}(F, x) \leq \varphi_{i}\left(F, x^{\prime}\right)\right.$ or $\left.\varphi_{j}(F, x) \leq \varphi_{j}\left(F, x^{\prime}\right)\right\}$.

Observe that this condition does not imply Monotonicity.

3) Finally, we repeat that the nearly serial methods form a very small class. In the continuous model (where input contributions are real numbers), these methods have no natural counterpart: we conjecture that the five axioms in Theorem 2 characterize precisely Friedman and Moulin's (1999) serial method. Friedman's (2004) representation theorem (stating that the methods meeting Additivity and Dummy are convex combinations of path methods) should prove useful in tackling this conjecture.

\section{APPENDIX}

We prove the "if" part of Theorem 2. The only statement that requires a proof is that all nearly serial methods satisfy Group Monotonicity. Let $N=\{1,2, \ldots, n\}, n \geq 3$, let $\varphi$ be a nearly serial method for $N$, and let $\bar{f}$ be the corresponding unbounded flow. This unbounded flow is anonymous and nearly diagonal. It is therefore fully characterized by a sequence of weights $\left\{\alpha_{r}\right\}$ as in (13). Let $F \in \mathcal{F}(N), S \subseteq N$, and let $x, x^{\prime} \in \mathbb{N}^{N}$ be such that $x_{i}<x_{i}^{\prime}$ for all $i \in S$ and $x_{i}=x_{i}^{\prime}$ for all $i \in N \backslash S$. Since it is well known that all fixed-flow methods satisfy Monotonicity, we may assume that $S$ contains at least two distinct agents. To shorten notation, write $f(., x)=g$ and $f\left(., x^{\prime}\right)=g^{\prime}$. These flows obtain by projecting $\bar{f}$ on $[0, x]$ and $\left[0, x^{\prime}\right]$, respectively:

$$
g=p_{[0, x]} \bar{f}
$$

and

$$
g^{\prime}=p_{\left[0, x^{\prime}\right]} \bar{f} .
$$

For ease of exposition, we give the argument for the case $n=3$ first. Without loss of generality, let $S=\{1,2\}$. Thus $x_{1}<x_{1}^{\prime}, x_{2}<x_{2}^{\prime}$ and $x_{3}=x_{3}^{\prime}$. Define $\bar{f}_{12}: \mathbb{N}^{N} \rightarrow \mathbb{R}^{\{1,2\}}$ by $\bar{f}_{12}(z)=\left(\bar{f}_{1}(z), \bar{f}_{2}(z)\right)$. Similarly, define $g_{12}=\left(g_{1}, g_{2}\right)$ on $[0, x]$ and $g_{12}^{\prime}=\left(g_{1}^{\prime}, g_{2}^{\prime}\right)$ on $\left[0, x^{\prime}\right]$. 
Case 1: $x_{1} \neq x_{2}$.

This is the easier case. Suppose that $x_{1}<x_{2}$. Because $\bar{f}$ is nearly diagonal, (26) and (27) give

$$
g_{1}(z)=g_{1}^{\prime}(z) \text { for all } z \in[0, x] .
$$

It follows that $\varphi_{1}(F, x) \leq \varphi_{1}\left(F, x^{\prime}\right)$. Permuting the roles of 1 and 2 , the same argument shows that $\varphi_{2}(F, x) \leq \varphi_{2}\left(F, x^{\prime}\right)$ when $x_{2}<x_{1}$.

Case 2: $x_{1}=x_{2}$.

For $r=0,1, \ldots, x_{3}$, define $Z(r)=\left\{z \in[0, x] \mid z_{3}=r\right\}$ and $Z^{\prime}(r)=\left\{z \in\left[0, x^{\prime}\right] \mid z_{3}=r\right\}$. For $i=1,2$, write

$$
\begin{aligned}
& \varphi_{i}(F, x)=\sum_{r=0}^{x_{3}} y_{i}(r), \\
& \varphi_{i}\left(F, x^{\prime}\right)=\sum_{r=0}^{x_{3}} y_{i}^{\prime}(r),
\end{aligned}
$$

where $y_{i}(r)=\sum_{z \in Z(r)} g_{i}(z) \partial_{i} F(z)$ and $y_{i}^{\prime}(r)=\sum_{z \in Z^{\prime}(r)} g_{i}^{\prime}(z) \partial_{i} F(z)$. In order to compare $\varphi_{i}(F, x)$ and $\varphi_{i}\left(F, x^{\prime}\right)$, we will compare $y_{i}(r)$ and $y_{i}^{\prime}(r)$ for each $r=0,1, \ldots, x_{3}$.

For any $r \in \mathbb{N}$, consider the restriction of $\bar{f}_{12}$ to $\bar{Z}(r)=\left\{z \in \mathbb{N}^{N} \mid z_{3}=r\right\}$. If $r \neq 0$, this mapping is fully determined by the three numbers $\alpha_{r-1}, \alpha_{r}, \alpha_{r+1}$ :

$$
\begin{aligned}
\bar{f}_{1}(r, r, r) & =\bar{f}_{1}(r+1, r, r)=\frac{\alpha_{r}}{3}, \\
\bar{f}_{1}(r, r-1, r) & =\bar{f}_{1}(r+1, r+1, r)=\frac{1}{6}, \\
\bar{f}_{1}(r-1, r-1, r) & =\frac{1-\alpha_{r-1}}{6}, \\
\bar{f}_{1}(r+1, r-1, r) & =\bar{f}_{1}(r, r+1, r)=\frac{1-\alpha_{r}}{6}, \\
\bar{f}_{1}(r+2, r+1, r) & =\frac{1-\alpha_{r+1}}{6}, \\
\bar{f}_{1}(., ., r) & =0 \text { otherwise }
\end{aligned}
$$

with the convention that $\alpha_{0}=1$, and $\bar{f}_{2}(., ., r)$ obtains by permutation. See Figure 14. The restriction of $\bar{f}_{12}$ to $\bar{Z}(0)$ is simply $\bar{f}_{1}(1,0,0)=\frac{1}{3}, \bar{f}_{1}(1,1,0)=\frac{1}{6}, \bar{f}_{1}(2,1,0)=\frac{1-\alpha_{1}}{6}$, $\bar{f}_{1}(., ., 0)=0$ otherwise, and $\bar{f}_{2}(., ., 0)$ obtains by permutation.

Case 2.1: $r<x_{3}$. In this case, the restriction of $g_{12}$ to $Z(r)$ and the restriction of $g_{12}^{\prime}$ to $Z^{\prime}(r)$ obtain by projecting the restriction of $\bar{f}_{12}$ to $\bar{Z}(r)$ on $Z(r)$ and $Z^{\prime}(r)$, respectively: $\left.g_{12}\right|_{Z(r)}=p_{Z(r)}\left(\left.\bar{f}_{12}\right|_{\bar{Z}(r)}\right)$ and $\left.g_{12}^{\prime}\right|_{Z^{\prime}(r)}=p_{Z^{\prime}(r)}\left(\left.\bar{f}_{12}\right|_{\bar{Z}(r)}\right)$.

If $r \neq x_{1}\left(=x_{2}\right)$, it is straightforward to check that $g_{12}$ and $g_{12}^{\prime}$ coincide in $Z(r)$. Formally, $\left[r \neq x_{1}\right] \Rightarrow\left[g_{12}(z)=g_{12}^{\prime}(z)\right.$ for all $\left.z \in Z(r)\right]$. It follows that

$$
y_{i}(r) \leq y_{i}^{\prime}(r) \text { for } i=1,2 \text {, for all } r<x_{3}, r \neq x_{1}=x_{2} .
$$

If $r=x_{1}\left(=x_{2}\right)$, the mappings $\left.g_{12}\right|_{Z(r)}$ and $\left.g_{12}^{\prime}\right|_{Z^{\prime}(r)}$ are illustrated in Figures 15.a and 15.b. This is the only delicate case. We claim that

$$
y_{i}(r) \leq y_{i}^{\prime}(r) \text { for some } i \in\{1,2\} \text {, for } r=x_{1}=x_{2}<x_{3} .
$$


To prove this, observe that

$$
\begin{aligned}
y_{1}(r)= & \frac{1-\alpha_{r-1}}{6} \partial_{1} F(r-1, r-1, r)+\frac{1}{6} \partial_{1} F(r, r-1, r)+\frac{1+\alpha_{r}}{6} \partial_{1} F(r, r, r), \\
y_{1}^{\prime}(r) \geq & \frac{1-\alpha_{r-1}}{6} \partial_{1} F(r-1, r-1, r)+\frac{1}{6} \partial_{1} F(r, r-1, r) \\
& +\frac{\alpha_{r}}{3} \partial_{1} F(r, r, r)+\frac{1-\alpha_{r}}{6} \partial_{1} F(r, r+1, r)+\frac{1-\alpha_{r}}{6} \partial_{1} F(r+1, r-1, r),
\end{aligned}
$$

and corresponding expressions for $y_{2}(r)$ and $y_{2}^{\prime}(r)$ obtain by permuting the first and second argument of the production function.

Taking differences,

$$
\begin{aligned}
& y_{1}^{\prime}(r)-y_{1}(r) \geq \frac{1-\alpha_{r}}{6}\left(\partial_{1} F(r, r+1, r)+\partial_{1} F(r+1, r-1, r)-\partial_{1} F(r, r, r)\right), \\
& y_{2}^{\prime}(r)-y_{2}(r) \geq \frac{1-\alpha_{r}}{6}\left(\partial_{2} F(r+1, r, r)+\partial_{2} F(r-1, r+1, r)-\partial_{2} F(r, r, r)\right) .
\end{aligned}
$$

Suppose now, contrary to the claim, that $y_{i}^{\prime}(r)<y_{i}(r)$ for $i=1,2$. Then

$$
\begin{aligned}
& \partial_{1} F(r, r, r)>\partial_{1} F(r, r+1, r)+\partial_{1} F(r+1, r-1, r) \text { and } \\
& \partial_{2} F(r, r, r)>\partial_{2} F(r+1, r, r)+\partial_{2} F(r-1, r+1, r) .
\end{aligned}
$$

But since $F$ is nondecreasing,

$$
\begin{aligned}
& \partial_{1} F(r, r, r) \leq \partial_{1} F(r, r+1, r)+\partial_{2} F(r-1, r+1, r) \text { and } \\
& \partial_{2} F(r, r, r) \leq \partial_{2} F(r+1, r, r)+\partial_{1} F(r+1, r-1, r),
\end{aligned}
$$

which are incompatible with the two strict inequalities just derived.

Case 2.2. $r=x_{3}$. The mappings $\left.g_{12}\right|_{Z\left(x_{3}\right)}$ and $\left.g_{12}^{\prime}\right|_{Z^{\prime}\left(x_{3}\right)}$ obtain by projecting on $Z\left(x_{3}\right)$ and $Z^{\prime}\left(x_{3}\right)$ the first two components of the unbounded flow $\bar{f}$ in or above $\bar{Z}\left(x_{3}\right)$. Letting $\overline{\bar{Z}}\left(x_{3}\right)=\left\{z \in \mathbb{N}^{N} \mid z_{3} \geq x_{3}\right\}$, we have $\left.g_{12}\right|_{Z\left(x_{3}\right)}=p_{Z\left(x_{3}\right)}\left(\left.\bar{f}_{12}\right|_{\bar{Z}\left(x_{3}\right)}\right)$ and $\left.g_{12}^{\prime}\right|_{Z^{\prime}\left(x_{3}\right)}=$ $p_{Z^{\prime}\left(x_{3}\right)}\left(\left.\bar{f}_{12}\right|_{\bar{Z}\left(x_{3}\right)}\right)$. This may be done in two steps. First we compute $p_{\bar{Z}\left(x_{3}\right)}\left(\left.\bar{f}_{12}\right|_{\bar{Z}\left(x_{3}\right)}\right)$. Denoting this mapping $h_{12}$, we find

$$
\begin{aligned}
h_{1}\left(x_{3}-1, x_{3}-1, x_{3}\right) & =\frac{1-\alpha_{x_{3}-1}}{6}, \\
h_{1}\left(x_{3}, x_{3}, x_{3}\right) & =\frac{1+\alpha_{x_{3}}}{6}, \\
h_{1}\left(x_{3}+m, x_{3}+m, x_{3}\right) & =\frac{\left.2+x_{1}+m+1, x_{3}+m, x_{3}\right)}{6} \text { for all } m \in \mathbb{N} \backslash\{0\}, \\
h_{1}\left(x_{3}, x_{3}+1, x_{3}\right) & =\frac{1-\alpha_{x_{3}}}{6}, \\
h_{1}\left(x_{3}+m, x_{3}+m+1, x_{3}\right) & =\frac{2-\alpha_{x_{3}+m}-\alpha_{x_{3}+m+1}}{6} \text { for all } m \in \mathbb{N} \backslash\{0\}, \\
h_{1}\left(x_{3}, x_{3}-1, x_{3}\right) & =\frac{1}{6},
\end{aligned}
$$




$$
\begin{aligned}
h_{1}\left(x_{3}+1, x_{3}, x_{3}\right) & =\frac{1+2 \alpha_{x_{3}}}{6}, \\
h_{1}\left(x_{3}+m+1, x_{3}+m-1, x_{3}\right) & =\frac{1-\alpha_{x_{3}+m}}{6}, \text { all } m \in \mathbb{N}, \\
h_{1}\left(., ., x_{3}\right) & =0 \text { otherwise, }
\end{aligned}
$$

and $h_{2}$ obtains by permutation. Then we project $h_{12}$ on $Z\left(x_{3}\right)$ and $Z^{\prime}\left(x_{3}\right)$ to obtain $\left.g_{12}\right|_{Z\left(x_{3}\right)}$ and $\left.g_{12}^{\prime}\right|_{Z^{\prime}\left(x_{3}\right)}$.

If $x_{3}>x_{1}\left(=x_{2}\right)$, it is straightforward to check that $g_{12}$ and $g_{12}^{\prime}$ coincide in $Z\left(x_{3}\right)$ so that

$$
y_{i}\left(x_{3}\right) \leq y_{i}^{\prime}\left(x_{3}\right) \text { for } i=1,2 \text { if } x_{3}>x_{1}\left(=x_{2}\right) .
$$

If $x_{3} \leq x_{1}\left(=x_{2}\right)$, the argument in case 2.1 can be mimicked to show that

$$
y_{i}\left(x_{3}\right) \leq y_{i}^{\prime}\left(x_{3}\right) \text { for some } i \in\{1,2\} \text {, if } x_{3} \leq x_{1}=x_{2} .
$$

Gathering (28) to (31) establishes that $\varphi_{i}(F, x) \leq \varphi_{i}\left(F, x^{\prime}\right)$ for some $i \in\{1,2\}$ when $x_{1}=x_{2}$ and completes the proof of Group Monotonicity when $n=3$.

To generalize the argument to any $n \geq 3$, define $T=\left\{i \in S \mid x_{i} \leq x_{j}\right.$ for all $\left.j \in S\right\}$.

Case 1: $T$ is a singleton, say $T=\{i\}$.

Because $\bar{f}$ is nearly diagonal, (26) and (27) give

$$
g_{i}(z)=g_{i}^{\prime}(z) \text { for all } z \in[0, x]
$$

and it follows that $\varphi_{i}(F, x) \leq \varphi_{i}\left(F, x^{\prime}\right)$.

Case 2: $T$ is not a singleton.

By definition, all agents in $T$ contribute the same number of units, say, $x_{i}=\rho$ for all $i \in T$. Define $\bar{f}_{T}: \mathbb{N}^{N} \rightarrow \mathbb{R}^{T}$ by $\bar{f}_{T}(z)=\left(\bar{f}_{i}(z)\right)_{i \in T}$. Similarly, define $g_{T}=\left(g_{i}\right)_{i \in T}$ on $[0, x]$ and $g_{T}^{\prime}=\left(g_{i}^{\prime}\right)_{i \in T}$ on $\left[0, x^{\prime}\right]$. For any $r_{N \backslash T} \in\left[0, x_{N \backslash T}\right]$, define $Z\left(r_{N \backslash T}\right)=\{z \in[0, x] \mid$ $\left.z_{N \backslash T}=r_{N \backslash T}\right\}$ and $Z^{\prime}\left(r_{N \backslash T}\right)=\left\{z \in\left[0, x^{\prime}\right] \mid z_{N \backslash T}=r_{N \backslash T}\right\}$. For $i \in T$,

$$
\begin{aligned}
\varphi_{i}(F, x) & =\sum_{r_{N \backslash T} \in\left[0, x_{N \backslash T}\right]} y_{i}\left(r_{N \backslash T}\right), \\
\varphi_{i}\left(F, x^{\prime}\right) & =\sum_{r_{N \backslash T} \in\left[0, x_{N \backslash T}^{\prime}\right]} y_{i}^{\prime}\left(r_{N \backslash T}\right) \\
& \geq \sum_{r_{N \backslash T} \in\left[0, x_{N \backslash T}\right]} y_{i}^{\prime}\left(r_{N \backslash T}\right),
\end{aligned}
$$

where $y_{i}\left(r_{N \backslash T}\right)=\sum_{z \in Z\left(r_{N \backslash T}\right)} g_{i}(z) \partial_{i} F(z)$ and $y_{i}^{\prime}\left(r_{N \backslash T}\right)=\sum_{z \in Z^{\prime}\left(r_{N \backslash T}\right)} g_{i}^{\prime}(z) \partial_{i} F(z)$. In order to compare $\varphi_{i}(F, x)$ and $\varphi_{i}\left(F, x^{\prime}\right)$, we will compare $y_{i}\left(r_{N \backslash T}\right)$ and $y_{i}^{\prime}\left(r_{N \backslash T}\right)$ for each $r_{N \backslash T} \in\left[0, x_{N \backslash T}\right]$.

Fix $r_{N \backslash T} \in\left[0, x_{N \backslash T}\right]$ and let $\bar{Z}\left(r_{N \backslash T}\right)=\left\{z \in \mathbb{N}^{N} \mid z_{N \backslash T}=r_{N \backslash T}\right\}$. Let $R_{1}=\{i \in N \backslash T \mid$ $\left.r_{i}<x_{i}\right\}, R_{2}=\left\{i \in N \backslash T \mid r_{i}=x_{i}\right\}$, and let $\overline{\bar{Z}}\left(r_{N \backslash T}\right)=\left\{z \in \mathbb{N}^{N} \mid z_{R_{1}}=r_{R_{1}}\right.$ and $\left.z_{R_{2}} \geq x_{R_{2}}\right\}$. Define $h: \bar{Z}\left(r_{N \backslash T}\right) \rightarrow \mathbb{R}_{+}^{T}$ by

$$
h=p_{\bar{Z}\left(r_{N \backslash T}\right)}\left(\left.\bar{f}_{T}\right|_{\overline{\bar{Z}}\left(r_{N \backslash T}\right)}\right) .
$$


By construction, this mapping is nearly diagonal with respect to $T$ : for all $z \in \bar{Z}\left(r_{N \backslash T}\right)$ and all $i \in T, h_{i}(z)=0$ whenever $z_{T} \notin \bar{D}_{T}$ or $z_{T}-e_{T}^{i} \notin \bar{D}_{T}$, where $\bar{D}_{T}$ denotes the near diagonal of $\mathbb{N}^{T}$. Moreover, $h$ is anonymous with respect to $T$ : for all $\left(z_{T}, r_{N \backslash T}\right) \in \bar{Z}\left(r_{N \backslash T}\right)$ and any permutation $\sigma$ on $T, h\left(\sigma z_{T}, r_{N \backslash T}\right)=\sigma h\left(z_{T}, r_{N \backslash T}\right)$. Finally, by (26) and (27),

$$
\left.g_{T}\right|_{Z\left(r_{N \backslash T}\right)}=p_{Z\left(r_{N \backslash T}\right)} h
$$

and

$$
\left.g_{T}^{\prime}\right|_{Z^{\prime}\left(r_{N \backslash T}\right)}=p_{Z^{\prime}\left(r_{N \backslash T}\right)} h .
$$

It is tedious but easy to verify that

$$
y_{i}\left(r_{N \backslash T}\right) \leq y_{i}^{\prime}\left(r_{N \backslash T}\right) \text { for all } i \in T \text {, for all } r_{N \backslash T} \neq \rho e_{N \backslash T}^{N} \wedge x_{N \backslash T},
$$

where $\left(z_{N \backslash T} \wedge z_{N \backslash T}^{\prime}\right)_{i}=\min \left\{z_{i}, z_{i}^{\prime}\right\}$ for all $i \in N \backslash T$. This follows directly from the projection formulas (32), (33) and the fact that $h$ is nearly diagonal with respect to $T$.

The more difficult case is when $r_{N \backslash T}=\rho e_{N \backslash T}^{N} \wedge x_{N \backslash T}$. We claim that

$$
y_{i}\left(\rho e_{N \backslash T}^{N} \wedge x_{N \backslash T}\right) \leq y_{i}^{\prime}\left(\rho e_{N \backslash T}^{N} \wedge x_{N \backslash T}\right) \text { for some } i \in T .
$$

Let us prove this claim for the case $\rho e_{N \backslash T}^{N} \ll x_{N \backslash T}$ first. The projection formulas (33), (34) yield

$$
\begin{aligned}
& y_{i}^{\prime}\left(\rho e_{N \backslash T}^{N}\right)-y_{i}\left(\rho e_{N \backslash T}^{N}\right) \\
\geq & \frac{1-\alpha_{\rho}}{n(n-1)} \sum_{j \in T \backslash i}\left[\partial_{i} F\left((\rho+1) e^{i}+(\rho-1) e^{j}+\rho e^{N \backslash i j}\right)\right. \\
& \left.+\partial_{i} F\left((\rho+1) e^{j}+\rho e^{N \backslash j}\right)-\partial_{i} F\left(\rho e^{N}\right)\right]
\end{aligned}
$$

where $n(n-1)$ is the number of permutations of the point $(\rho-1) e^{1}+\rho e^{\{2, \ldots, n-1\}}+(\rho+1) e^{n}$.

Suppose, by contradiction, that $y_{i}^{\prime}\left(\rho e_{N \backslash T}^{N}\right)<y_{i}\left(\rho e_{N \backslash T}^{N}\right)$ for all $i \in T$. Then $\sum_{j \in T \backslash i} \partial_{i} F\left(\rho e^{N}\right)$ $>\sum_{j \in T \backslash i}\left[\partial_{i} F\left((\rho+1) e^{i}+(\rho-1) e^{j}+\rho e^{N \backslash i j}\right)+\partial_{i} F\left((\rho+1) e^{j}+\rho e^{N \backslash j}\right)\right]$ for all $i \in T$. Summing up these inequalities,

$$
\begin{aligned}
& \sum_{i \in T} \sum_{j \in T \backslash i} \partial_{i} F\left(\rho e^{N}\right) \\
> & \sum_{i \in T} \sum_{j \in T \backslash i}\left[\partial_{i} F\left((\rho+1) e^{i}+(\rho-1) e^{j}+\rho e^{N \backslash i j}\right)+\partial_{i} F\left((\rho+1) e^{j}+\rho e^{N \backslash j}\right)\right] \\
= & \sum_{i \in T} \sum_{j \in T \backslash i}\left[\partial_{j} F\left((\rho-1) e^{i}+(\rho+1) e^{j}+\rho e^{N \backslash i j}\right)+\partial_{i} F\left((\rho+1) e^{j}+\rho e^{N \backslash j}\right)\right] .
\end{aligned}
$$

But since $F$ is nondecreasing,

$$
\partial_{i} F\left(\rho e^{N}\right) \leq \partial_{j} F\left((\rho-1) e^{i}+(\rho+1) e^{j}+\rho e^{N \backslash i j}\right)+\partial_{i} F\left((\rho+1) e^{j}+\rho e^{N \backslash j}\right)
$$

for all $i \in T, j \in T \backslash i$, a contradiction.

Dispensing now with the assumption $\rho e_{N \backslash T}^{N} \ll x_{N \backslash T}$, let $r_{N \backslash T}=\rho e_{N \backslash T}^{N} \wedge x_{N \backslash T}$. Because $h$ is nearly diagonal and anonymous with respect to $T$, we obtain

$$
\begin{aligned}
& y_{i}^{\prime}\left(r_{N \backslash T}\right)-y_{i}\left(r_{N \backslash T}\right) \\
\geq & A_{\rho} \sum_{j \in T \backslash i}\left[\partial_{i} F\left((\rho+1) e_{T}^{i}+(\rho-1) e_{T}^{j}+\rho e_{T}^{N \backslash i j}, r_{N \backslash T}\right)\right. \\
& \left.+\partial_{i} F\left((\rho+1) e_{T}^{j}+\rho e_{T}^{N \backslash j}, r_{N \backslash T}\right)-\partial_{i} F\left(\rho e_{T}^{N}, r_{N \backslash T}\right)\right],
\end{aligned}
$$


where $A_{\rho}$ is a coefficient that does not depend on $i$. If $y_{i}^{\prime}\left(r_{N \backslash T}\right)<y_{i}\left(r_{N \backslash T}\right)$ for all $i \in T$, a contradiction is obtained just as before.

\section{REFERENCES}

[1] Aumann, R.J., and Shapley, L. (1974). Values of Nonatomic Games. Princeton: Princeton Univ. Press.

[2] Billera, L., and Heath, D. (1982). "Allocation of Shared Costs: A Set of Axioms Yielding a Unique Procedure," Math. Oper. Res. 7, 32-39.

[3] Friedman, E. (2004), "Paths and Consistency in Additive Cost Sharing," Int. J. Game Theory 32, 501-518.

[4] Friedman, E., Moulin, H. (1999). "Three Methods to Share Joint Costs or Surplus," J. Econ. Theory 87, 275-312.

[5] Mirman, L., and Tauman, Y. (1982). "Demand Compatible Equitable Cost Sharing Prices," Math. Oper. Res. 7, 40-56.

[6] Moulin, H. (1995). "On Additive Methods to Share Joint Costs," Japan Econ. Rev. 46, 303-332.

[7] Moulin, H. (2002). "Axiomatic Cost and Surplus Sharing," in Handbook of Social Choice and Welfare (Arrow, K.J., Sen, A., and Suzumura, K., eds.), Amsterdam: Elsevier, 289-357.

[8] Moulin, H., and Shenker, S. (1992). "Serial Cost Sharing," Econometrica 60, 10091037.

[9] Moulin, H., and Shenker, S. (1994). "Average Cost Pricing versus Serial Cost Sharing: An axiomatic comparison," J. Econ. Theory 64, 178-201.

[10] Moulin, H., and Sprumont, Y. (2003). "Responsibility and Cross-Subsidization in Cost Sharing," mimeo, Rice University.

[11] Moulin, H., and Sprumont, Y. (2004). "On Demand Responsiveness in Additive Cost Sharing," mimeo, Rice University, forthcoming in J. Econ. Theory.

[12] Moulin, H., and Vohra, R. (2003). "A Representation of Additive Cost Sharing Methods," Econ. Letters 80, 399-407.

[13] Shapley, L. (1953). "A Value for $n$-Person Games," in Contributions to the Theory of Games II (Kuhn, H.W., and Tucker, W., eds.), Ann. Math. Stud. Vol. 28, NJ: Princeton Univ. Press.

[14] Shubik, M. (1962). "Incentives, Decentralized Control, the Assignment of Joint Costs, and Internal Pricing," Manage. Sci. 8, 325-343. 
Figure 1

$D$ and $\bar{D}$ when $n=2$

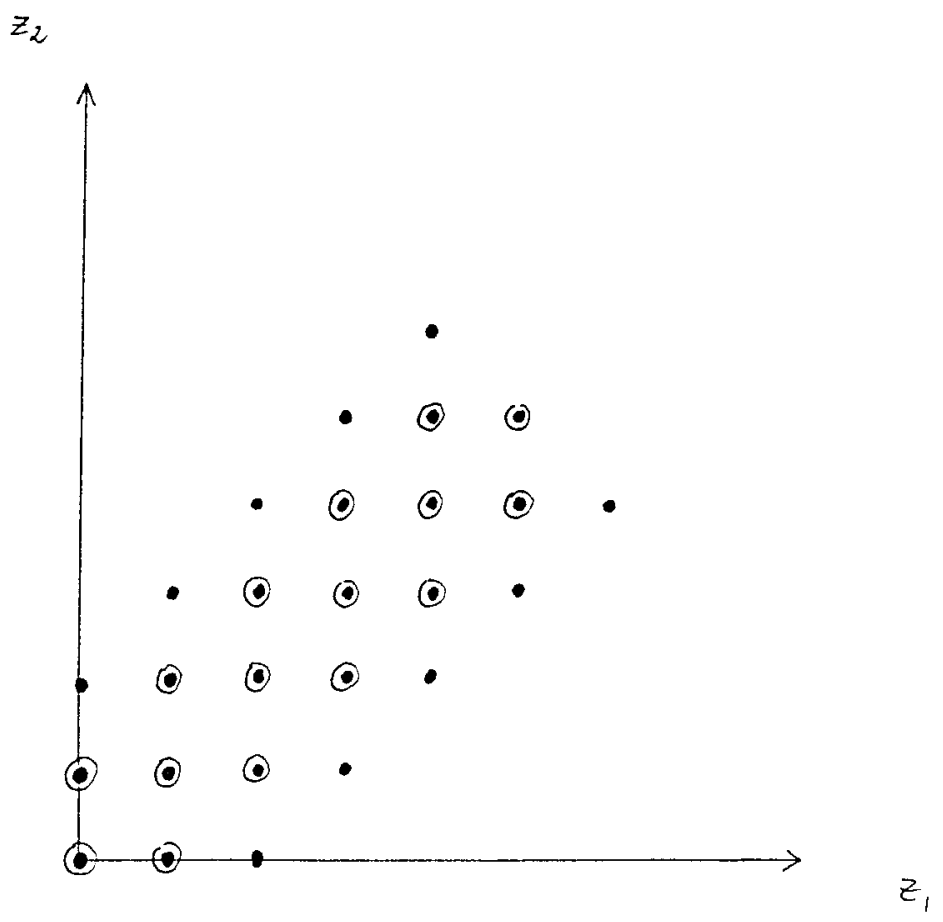

Figure 2

$D(t)$ and $\bar{D}(t)$ for $t=1,2,3,4$ when $n=3$
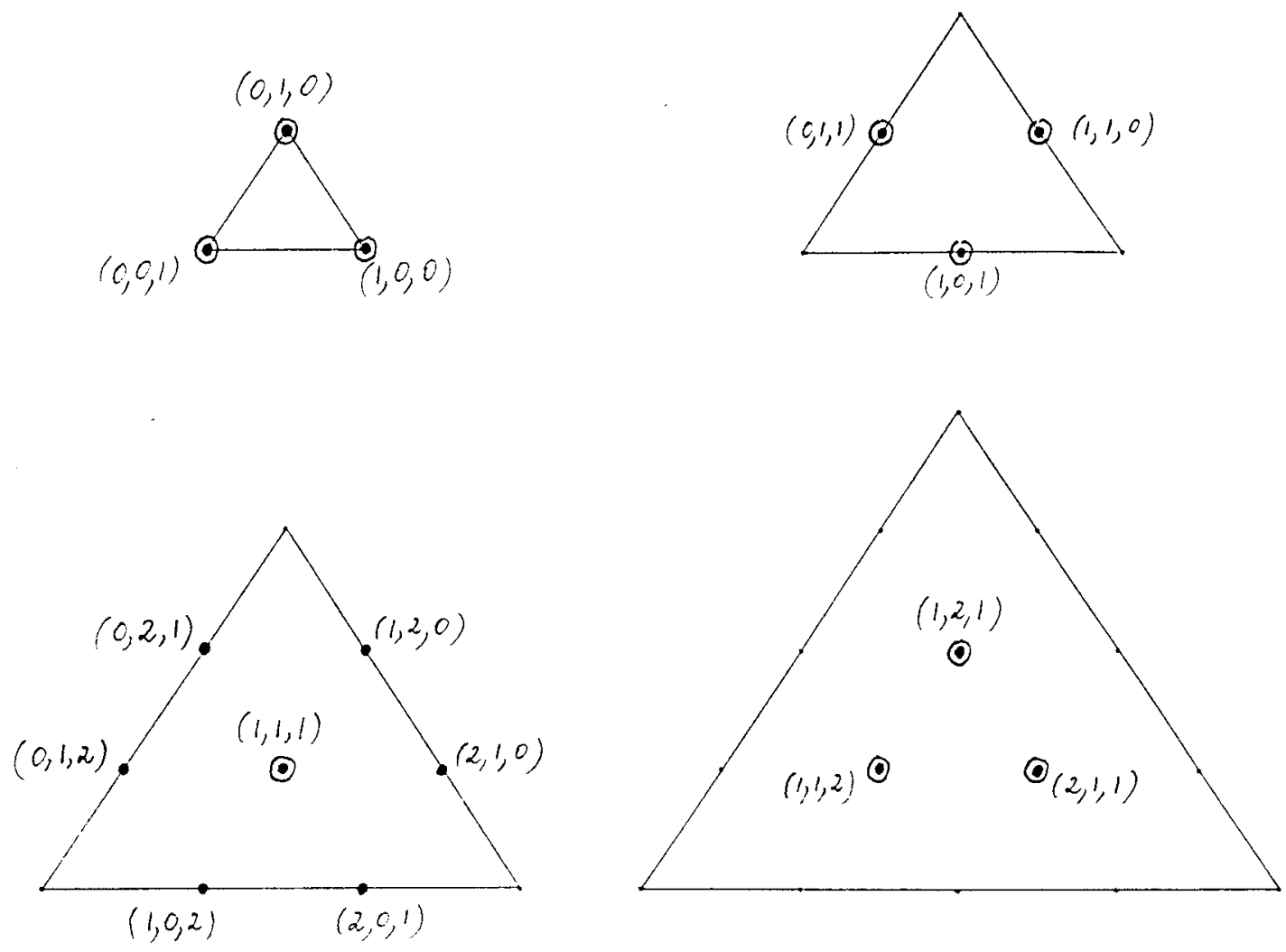
Figure 3

The flow $f^{S}(,,(1,1,1))$
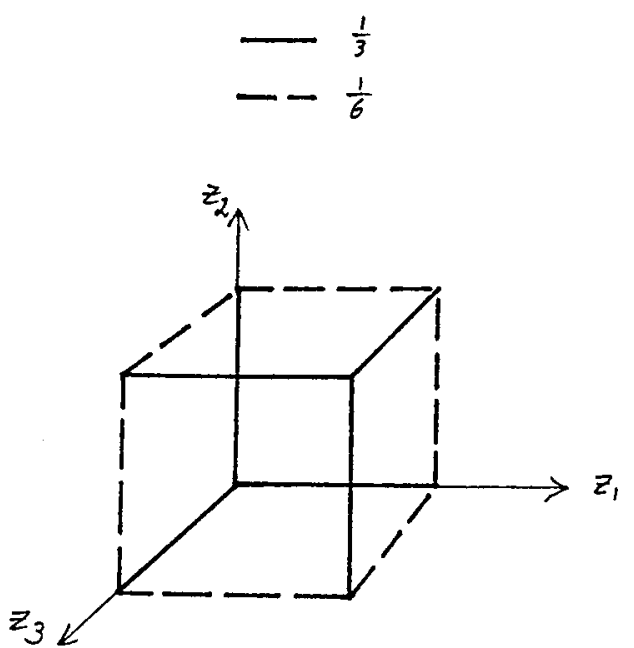

Figure 4

The flow $f^{s}(.,(2,2,2))$
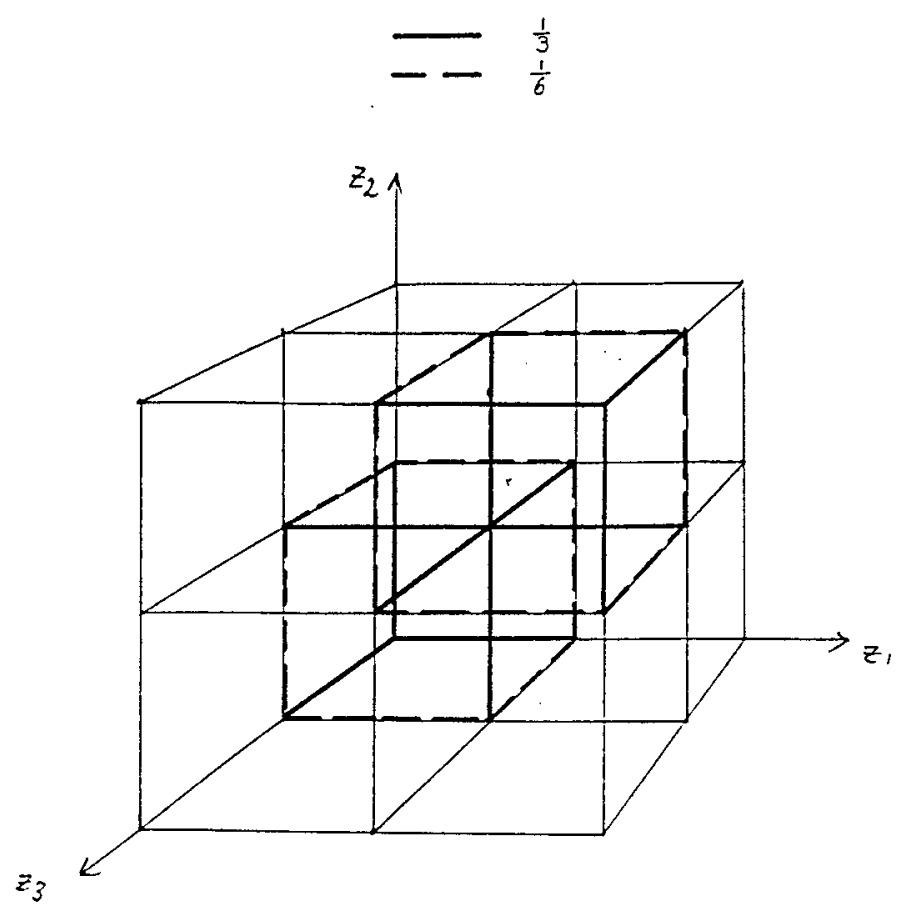
Figure 5

An anonymous nearly diagonal unbounded flow when $n=2$

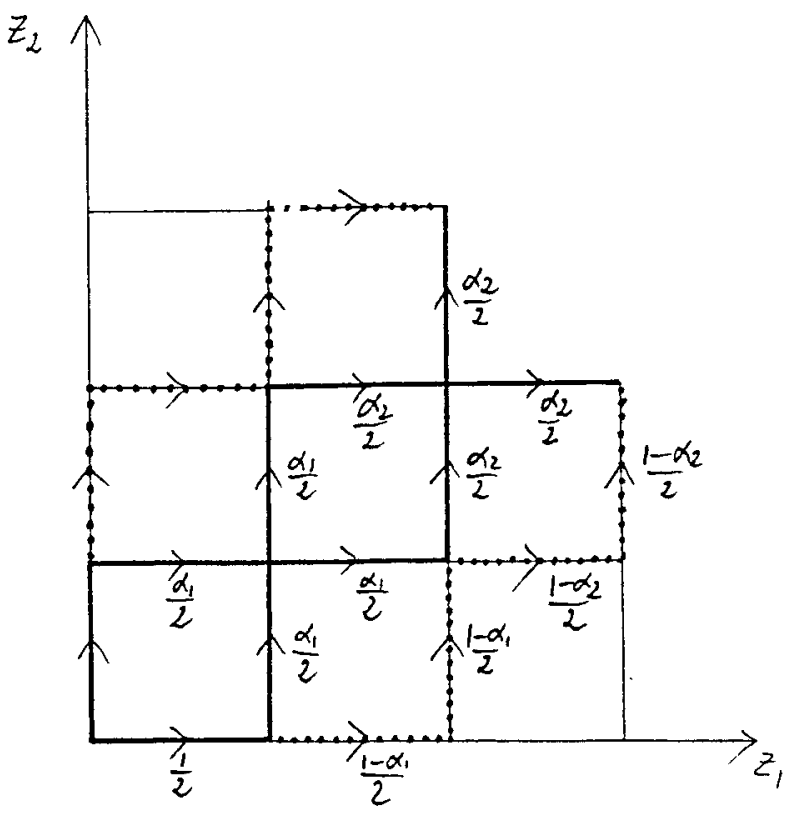

Figure 6.a

An anonymous nearly diagonal unbounded flow (at $\bar{D}(3), \bar{D}(4), \bar{D}(5)$ ) when $n=3$

$$
-\cdots \frac{\frac{\alpha_{1}}{3}}{\frac{1-\alpha_{1}}{6}}
$$

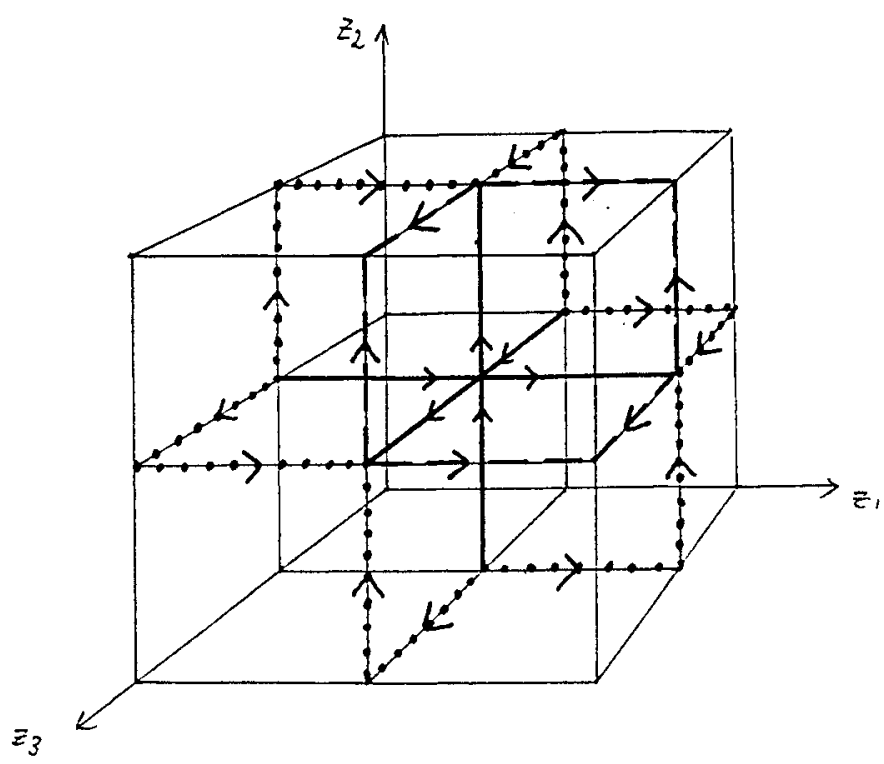



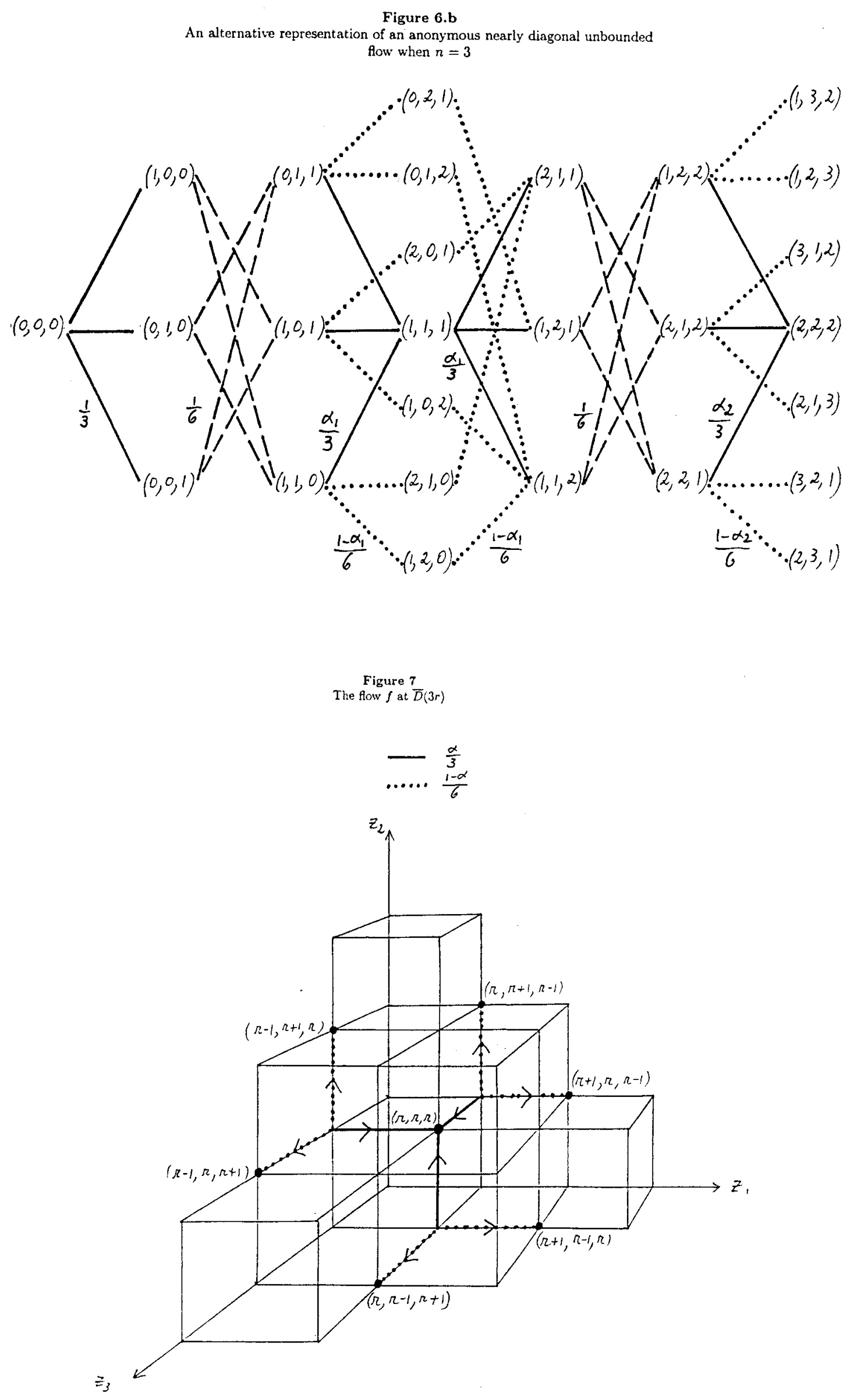


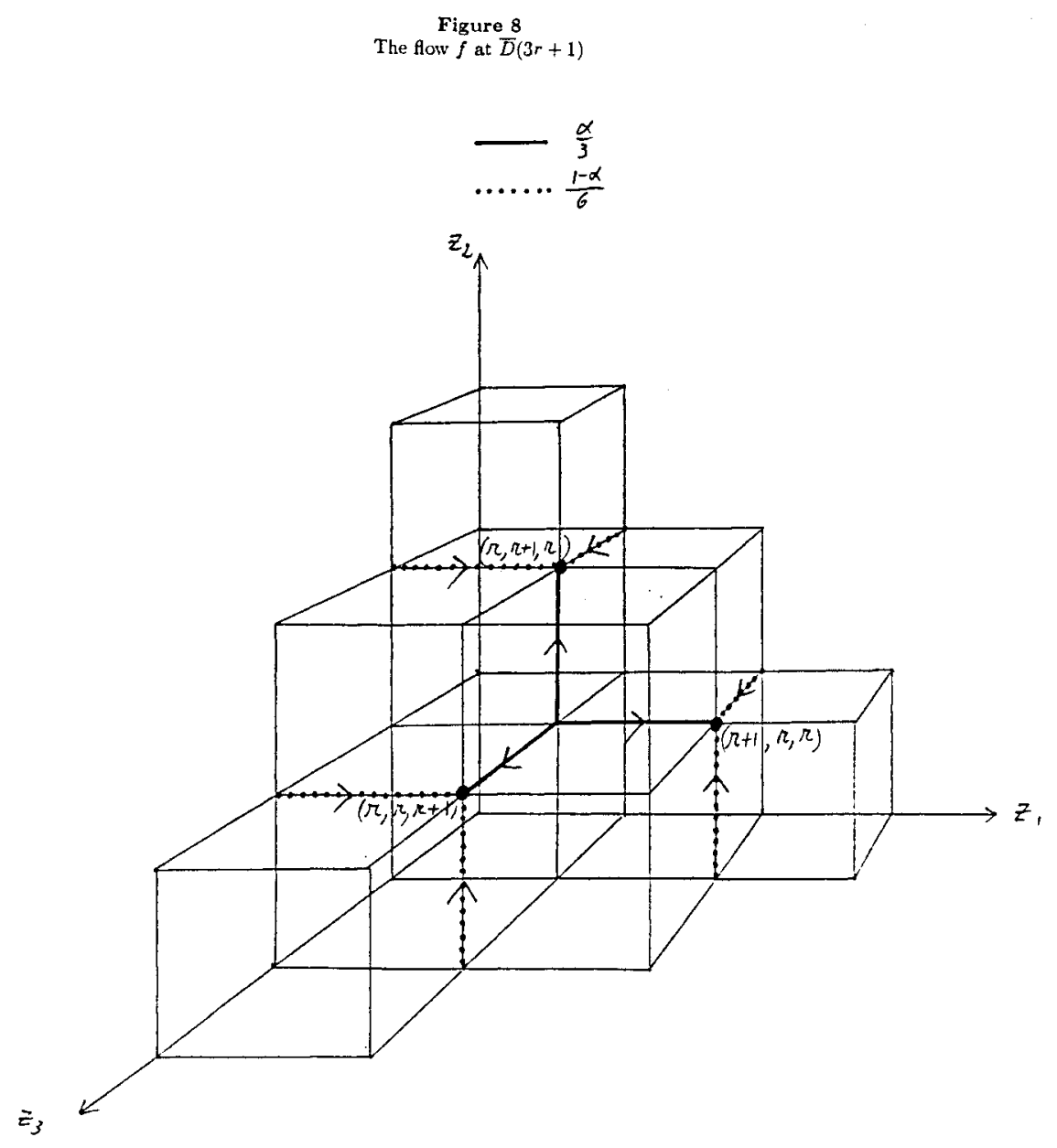

Figure 9

The flow $g$ and the lower frontier of $F$

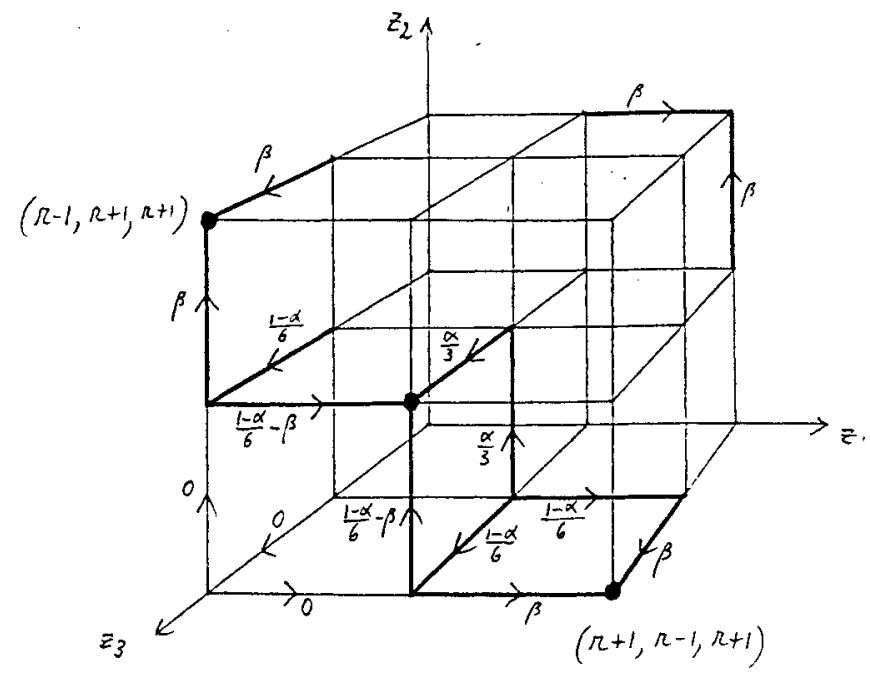


Figure 10

The lower frontier of $F^{\prime}$

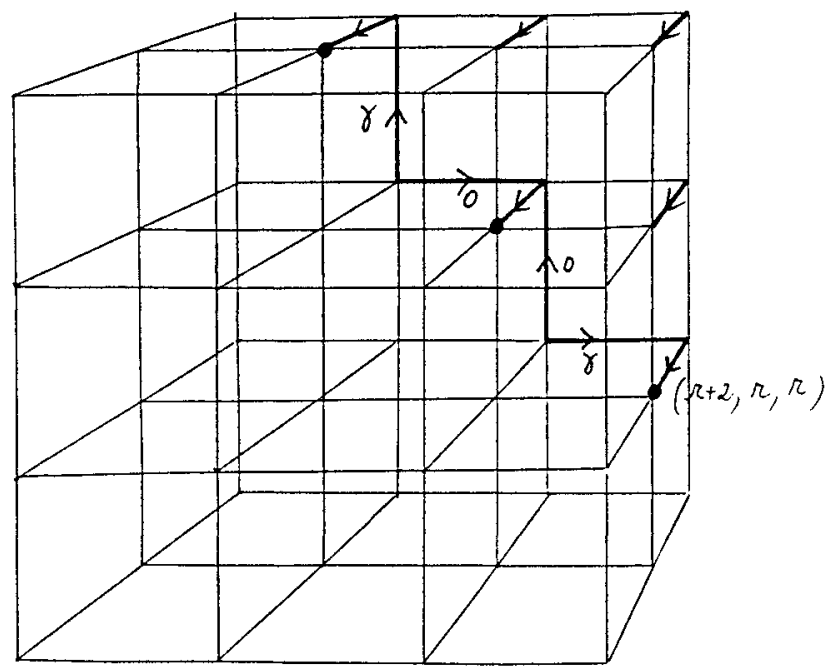

Figure 11

The lower frontier of $F^{\prime \prime}$

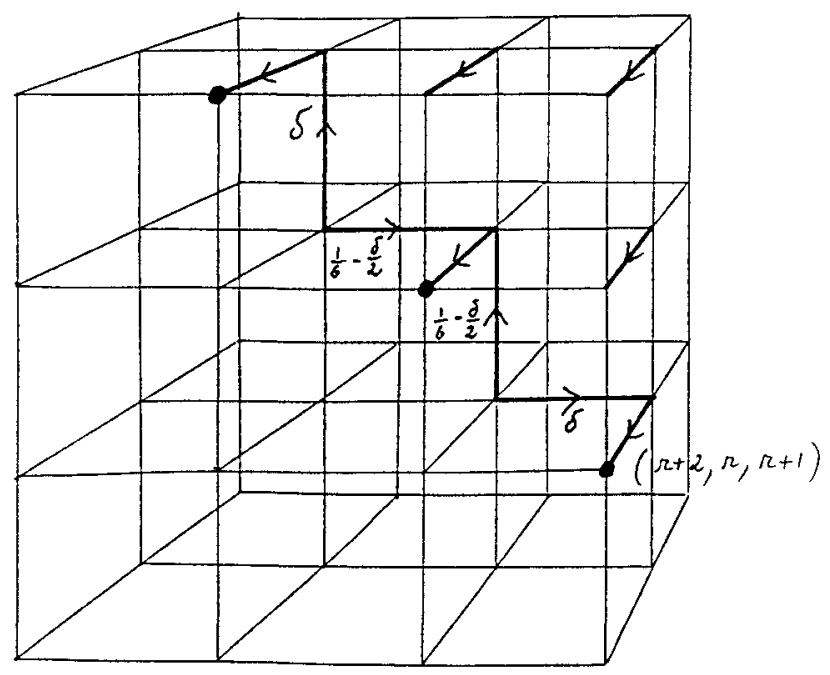


Figure 12

The fow $f$ at $\bar{D}(3 r+2)$

$-\frac{1}{6}$

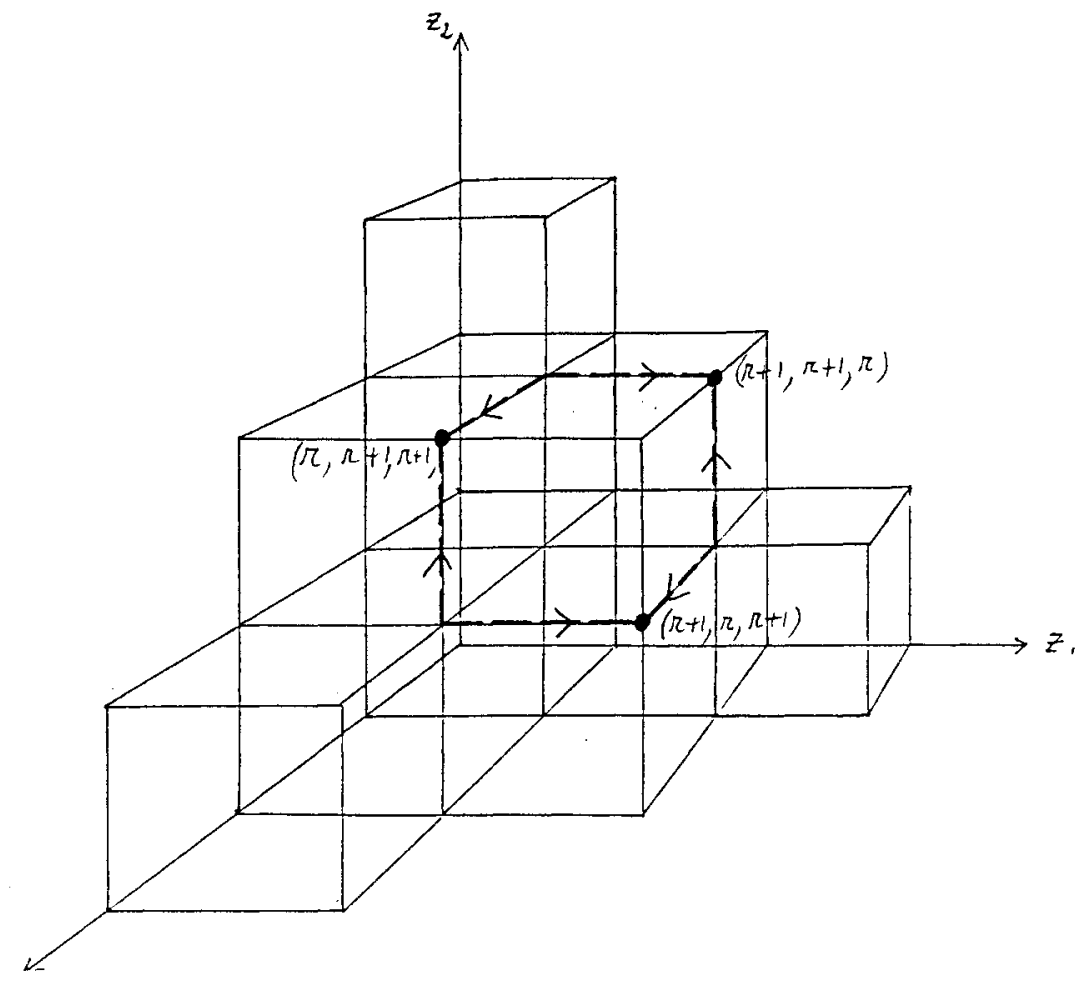

Figure 13
The flow $f$ at $\bar{D}(3 r-1)$
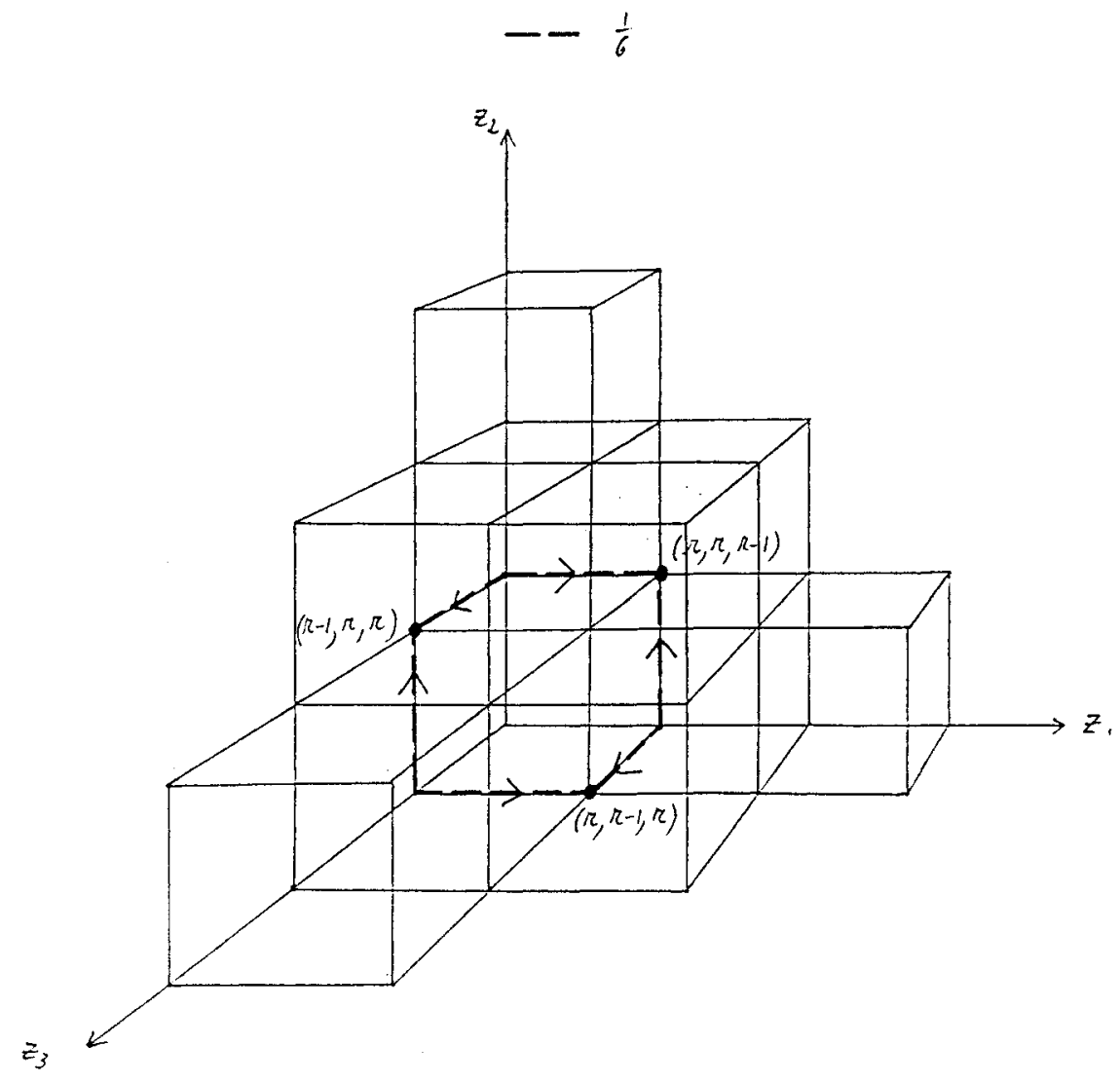

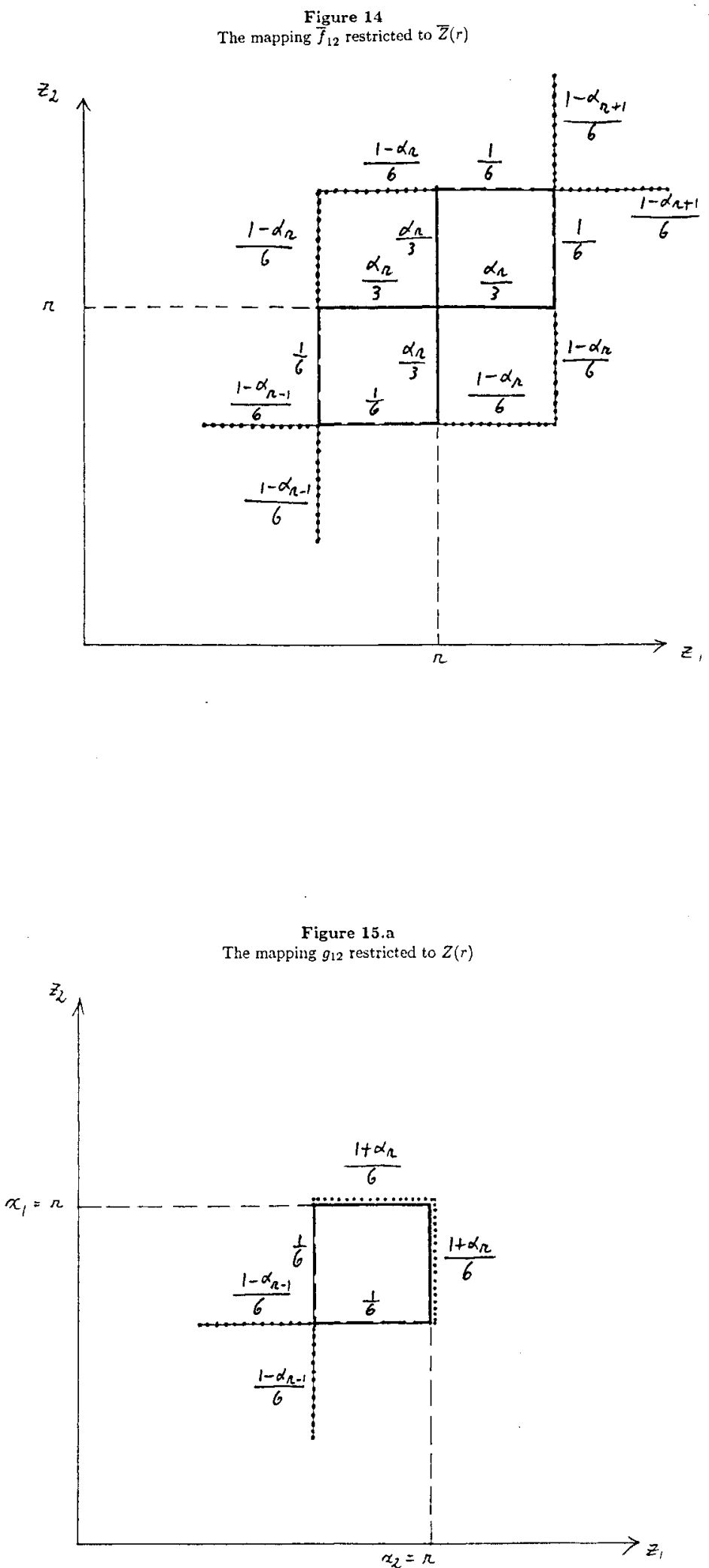
Figure 15.b

The mapping $g_{12}^{\prime}$ restricted to $Z^{\prime}(r)$

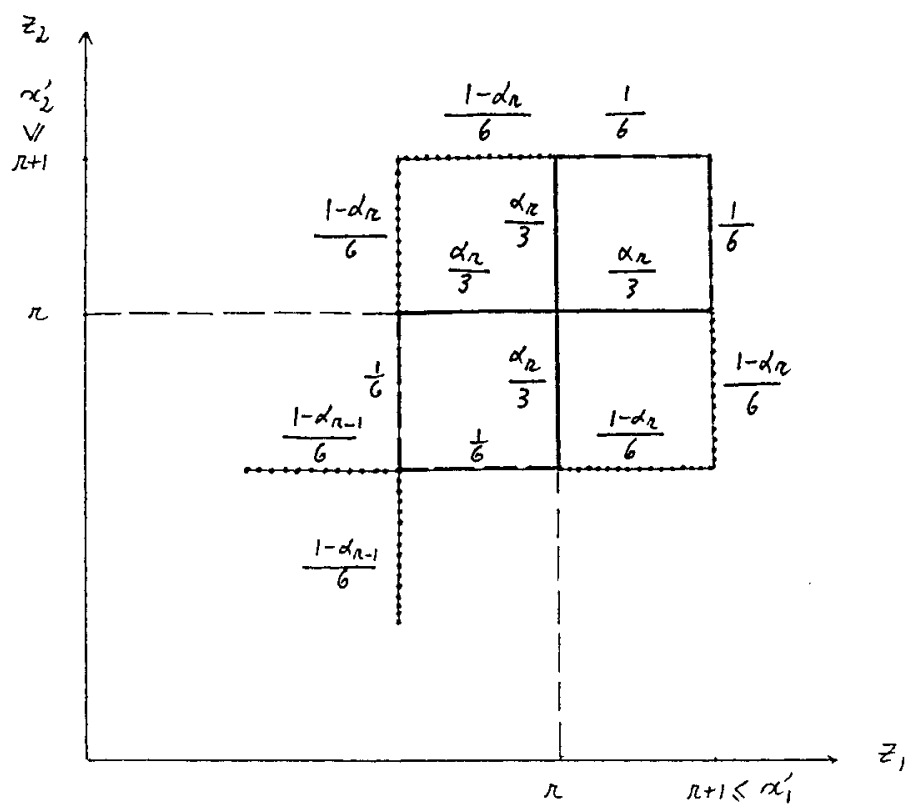

